Florida International University FIU Digital Commons

FIU Electronic Theses and Dissertations

University Graduate School

$11-17-2017$

\title{
CFD Evaluation of Mixing Processes for High- Level Nuclear Waste Tanks
}

Maximiliano Edrei

Florida International University, medre001@fiu.edu

DOI: 10.25148 /etd.FIDC004038

Follow this and additional works at: https://digitalcommons.fiu.edu/etd

Part of the Mechanical Engineering Commons

\section{Recommended Citation}

Edrei, Maximiliano, "CFD Evaluation of Mixing Processes for High-Level Nuclear Waste Tanks" (2017). FIU Electronic Theses and Dissertations. 3528.

https://digitalcommons.fiu.edu/etd/3528

This work is brought to you for free and open access by the University Graduate School at FIU Digital Commons. It has been accepted for inclusion in FIU Electronic Theses and Dissertations by an authorized administrator of FIU Digital Commons. For more information, please contact dcc@fiu.edu. 


\section{FLORIDA INTERNATIONAL UNIVERSITY}

Miami, Florida

CFD EVALUATION OF MIXING PROCESSES FOR HIGH-LEVEL NUCLEAR WASTE TANKS

A thesis submitted in partial fulfillment of the

Requirements for the degree of

MASTER OF SCIENCE

In

MECHANICAL ENGINEERING

by

Maximiliano Edrei

2017 
To: Dean John L. Volakis

College of Engineering and Computing

This thesis, written by Maximiliano Edrei, and entitled CFD Evaluation of Mixing Processes for High-Level Nuclear Waste Tanks, having been approved in respect to style and intellectual content, is referred to you for judgment.

We have read this thesis and recommend that it be approved.

Leonel Lagos

Dwayne McDaniel

Pezhman Mardanpour

Cheng-Xian Lin, Major Professor

Date of Defense: November 17, 2017

The thesis of Maximiliano Edrei is approved.

Dean John L. Volakis

College of Engineering and Computing

Andrés G. Gil

Vice President for Research and Economic Development and Dean of the University Graduate School

Florida International University, 2017 


\section{ACKNOWLEDGMENTS}

I would like to acknowledge and sincerely thank all who contributed to the work presented in this thesis. Specifically I would like to thank my advisors at the Applied Research Center Dr. Dwayne McDaniel and Dr. Leonel Lagos. Dr. Dwayne's mentoring went beyond the technical subject of this work and always paved a clear path for me to take, for which I am very thankful of. Dr. Lagos's involvement made all aspects of this work possible. I greatly appreciate the technical support I received from numerous faculty at NETL in Morgantown WV, more specifically that of Dr. Rahul Garg, Dr. Pankaj Sahaj, Balaji Gopalan, and Dr. Chris Guenther. Furthermore I would like to thank my major advisor Dr. Cheng-Xian Lin and research assistant Soheil Solemanikutanai. Without their support this work would not have gotten as far as it did. Finally I would like to thank Dr. Pezman Mandapour for providing useful perspective. 


\section{ABSTRACT OF THE THESIS \\ CFD EVALUATION OF MIXING PROCESSES FOR HIGH-LEVEL NUCLEAR WASTE TANKS \\ by \\ Maximiliano Edrei}

Florida International University, 2017

Miami, Florida

Professor Cheng-Xian Lin, Major Professor

Computational Fluid Dynamics (CFD) has been applied to investigate two aspects of a mixing process for high level nuclear waste tanks. Through CFD the applicability of Poreh's correlations that are currently used to describe the radial wall jets in the Pulse Jet Mixing (PJM) process were assessed. In addition, simulations were conducted in order to investigate mean hydrodynamic characteristics of sparged non-Newtonian fluids for the use in the PJM process.

Three single phase turbulent simulations using the commercial package STAR$\mathrm{CCM}+$ were successively conducted. A model validated with experimental data was developed and successively altered to see the effects of low characteristic ratio and a curved impingement surface. Results suggested that Poreh's correlations are applicable under PJM conditions and geometry.

Lastly, multi-phase Eulerian-Eulerian Simulations were conducted using the commercial software package ANSYS Fluent. Altering the characteristic ratio $(\mathrm{h} / \mathrm{D})$ of a sparged non-Newtonian fluid system resulted in a trend of flattening air volume fraction and air axial velocity profiles with decreasing characteristic ratio. 


\section{TABLE OF CONTENTS}

CHAPTER

PAGE

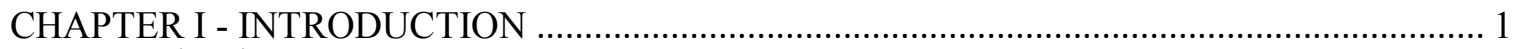

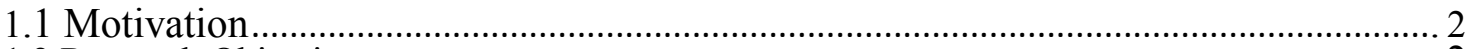

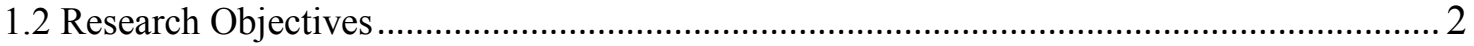

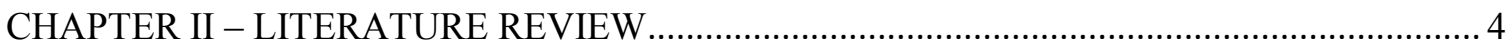

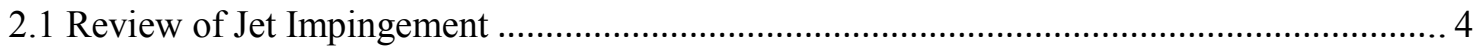

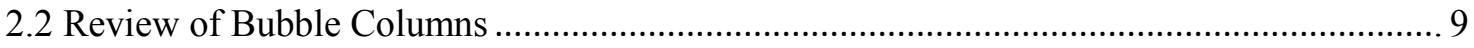

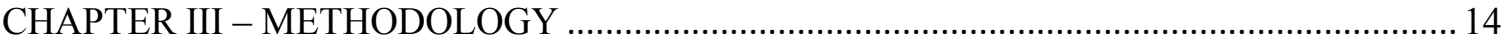

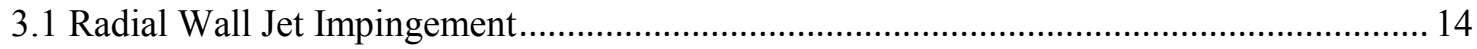

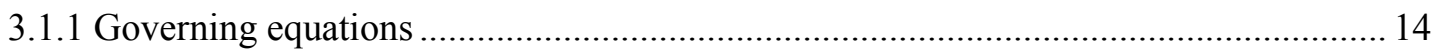

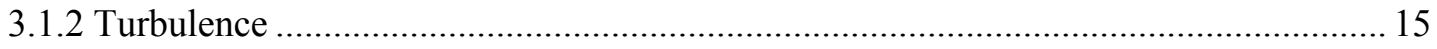

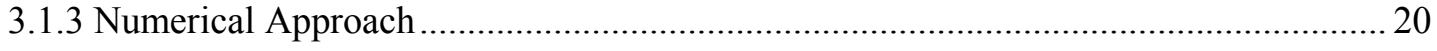

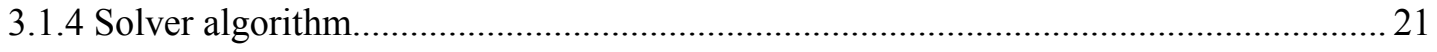

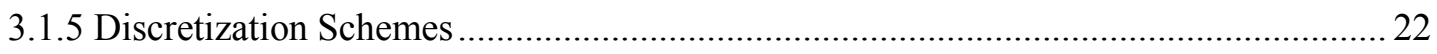

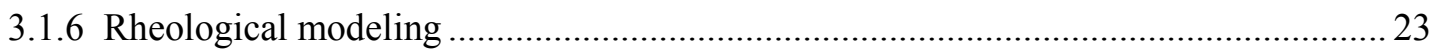

3.2 Sparging of Non-Newtonian Fluids in Bubble Columns ................................................ 23

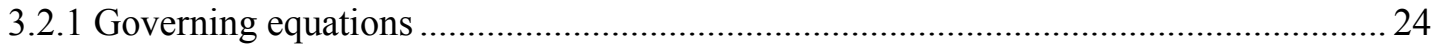

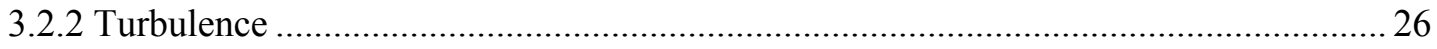

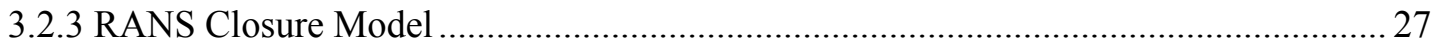

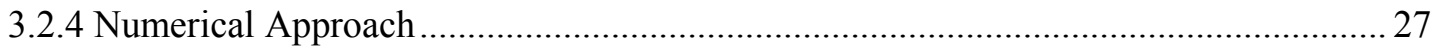

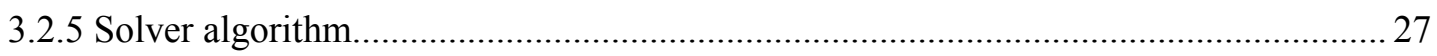

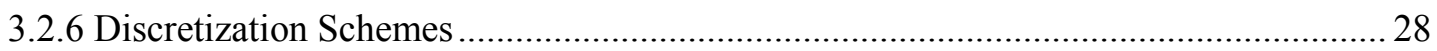

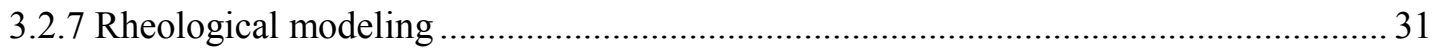

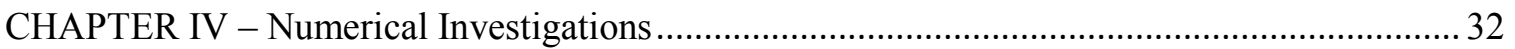

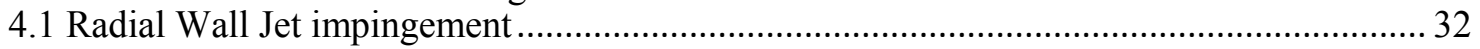

4.1.1 Replication of Poreh's Experiment and Reduction of Aspect Ratio (b/D) ................... 32

4.1.2 Curved Impingement Simulation of a Scaled Down PJM Model ( $\mathrm{b} / \mathrm{D}=1.5)$............... 39

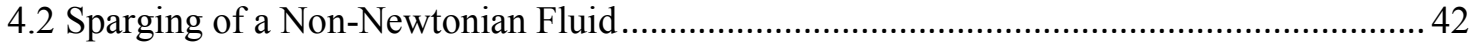

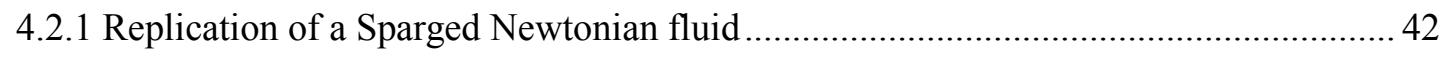

4.2.2 Simulation of Bubble column operating with Non-Newtonian Fluid ............................ 45

4.2.3 Parametric study Simulation of Bubble column operating with Non-Newtonian Fluid49

CHAPTER V - CONCLUSIONS AND FUTURE CONSIDERATION .................................... 56

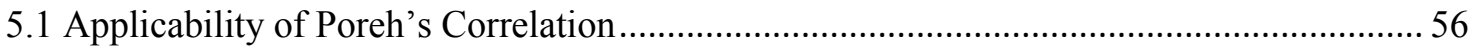

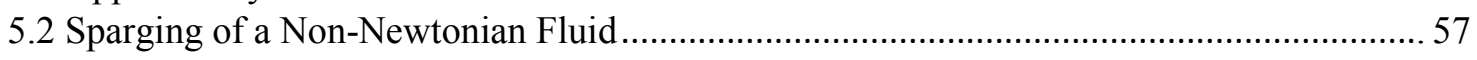

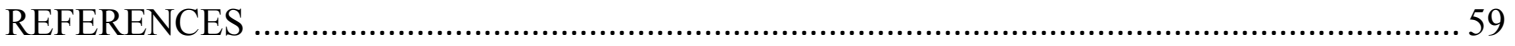




\section{LIST OF FIGURES}

FIGURE

PAGE

Figure 1-General flow structure in PJM vessel (Left), radial wall jet depiction (Poreh et al. 1967) (Right) .1

Figure 2-Bubble column flow regime based on characteristic length and flow rate .10

Figure 3- Reynolds decomposition .17

Figure 4- 1 Dimensional control volume. .29

Figure 5- Cell centroid .30

Figure 6- Schematic of computational domain showing geometrical dimensions, boundary conditions, and data extraction lines for (a) Poreh's experiment (b) and altered simulation reflecting lower $\mathrm{b} / \mathrm{d}$ ratio

Figure 7- Grid sensitivity test on mean velocity profiles (a) Circular Jet region (b) Radial wall jet region at $r b=1$ and (c) Radial wall jet region at $r b=2$

Figure 8-Computational mesh showing polyhedral mesh properties (a) $b d=12$ (b) $b d=1.5$

Figure 9- Comparison of wall-normal distribution of radial wall jet velocity profiles at radial locations; (normalized scale) (a) $r b=1 \quad$ (b) $r b=1.5 \quad$ (c) $r b=2$ $r b=2.5$ Figure $10-b D=12$ Simulation Comparison of radial variation $(r b)$ of (a) non-
dimensional radial wall jet maximum $U m b K$ and (b) non-dimensional radial wall jet thickness $\delta b$ at four radial locations

Figure $11-b D=1.5$ Simulation Comparison of radial variation $(r b)$ of (a) nondimensional radial wall jet maximum $U m b K$ and (b) non-dimensional radial wall jet thickness $\delta b$ at four radial locations.....

Figure 12- Schematic of PJM geometry and computational domain showing the geometrical dimensions with associated boundary conditions and the probe locations of radial velocity data extraction (a) 3D-quarter scaled down PJM vessel geometry (b) 2D 
plane of symmetry on which data was sampled (c) Cross-section view of polyhedral mesh......

Figure 13- $b D=1.5$ Curved impingement Simulation Comparison of radial variation ( $r b$ ) of (a) non-dimensional radial wall jet maximum $U m b K$ and (b) nondimensional radial wall jet thickness $\delta b$ at four radial locations

Figure 14- Boundary Conditions, physical characteristic (Left) and Initial conditions (Right) of Caixia Chen simulation...

Figure 15- Replication of Xe's simulation results compared to chen's experimental data 44

Figure 16- Cross section view of mesh for parametric study

Figure 17- Circumferentially time averaged (a) air volume fraction and (b) air axial velocity profile simulation results compared to Amin's experimental data .46

Figure 18- Developed simulation grid refinement study of the time averaged (a) air volume fraction (b) air axial velocity.....

Figure 19- Instantaneous velocity at the center of the bubble column at a height of (a) $40 \%$ of liquid height and (b) $80 \%$ of liquid height

Figure 20- H/D comparison ( $H D=5.2-.34$ ) of (a) air volume fraction and (b) air axial velocity at $40 \%$ of the liquid height

Figure 21- H/D comparison ( $H D=5.2-.34$ ) of (a) air volume fraction and (b) air axial velocity at $80 \%$ of the liquid height .50

Figure 22- Time averaged air volume fraction profile across a $2 \mathrm{D}$ plane for $\mathrm{H} / \mathrm{D}=5.8-.34$ with a clip to range of .5 (not to scale)

Figure 23- Time averaged air volume fraction on an axial section view for (a) $H D=.34$, (b) $H D=3.8$, (c) $H D=5.8$ at (1) $40 \%$ and (2) $80 \%$ of the liquid height .53 


\section{CHAPTER I - INTRODUCTION}

Currently, there are 53 million gallons of high level waste (HLW) being stored inside tanks located at the Hanford Site in Washington State. One of the Department of Energy's (DOE) primary objectives is to immobilize the waste in order to prevent contamination to the environment. The planned solution for this objective is to have the radioactive waste undergo separation and vitrification, which converts the waste into glass for permanent storage. The waste needs to have particular rheological properties before it goes through this process, including density, viscosity, porosity, etc. Within the tanks, the density of the HLW is not homogeneous. The HLW separates into multiple layers, referred to as salt cake, supernatant, and sludge, due to density differences. This creates the need to properly mix the HLW inside the storage tanks prior to treatment.

Pulse jet mixing (PJM), as shown on the left of Figure 1, is one of the methods selected by the DOE to mix the HLW slurry prior to the vitrification process. This method involves sucking a portion of the waste from the tank into a pressurized vessel and then injecting it back into the tank in order to mix the waste using pressurized jets. This process is repeated over a number of cycles until the desired level of homogeneity is achieved [1].
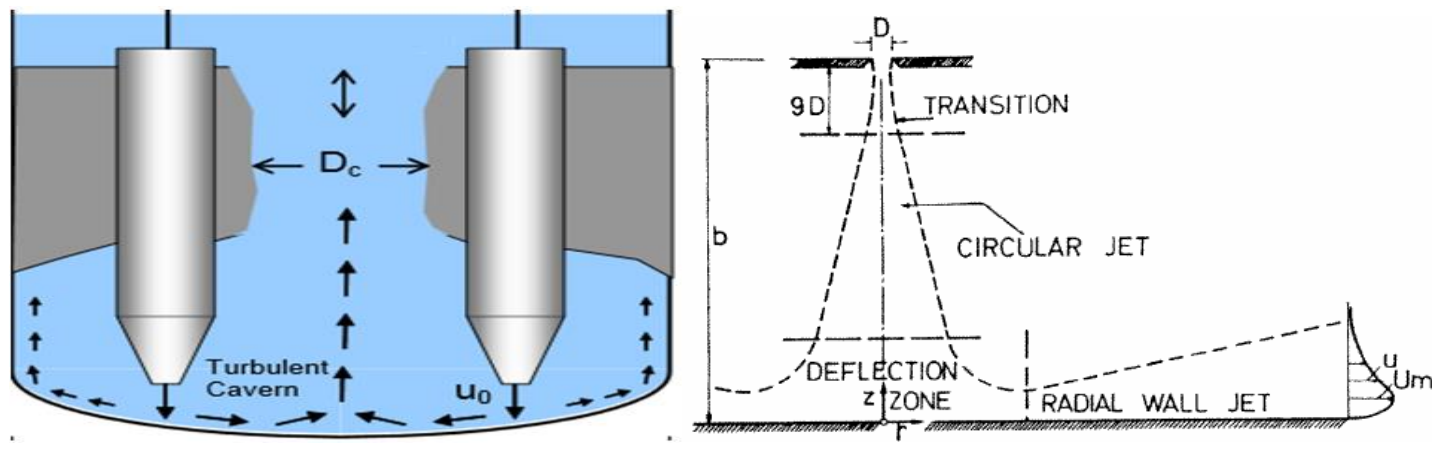

Figure 1-General flow structure in PJM vessel (Left), radial wall jet depiction (Poreh et al. 1967) (Right) 


\subsection{Motivation}

Currently, the PJM process as applied to the nuclear waste at Hanford is undergoing research and validation. For example, the analytical work describing the radial wall jets at the bottom of the PJM vessel, as depicted on the left of Figure 1, uses correlations developed by Poreh et al. [2]. Poreh's experimental investigation looked at the local maximum of radial wall jet velocity $\left(U_{m}\right)$ and the local characteristic jet thickness $(\delta)$ for a range of aspect ratios $(b / D)$ larger than those seen in the current PJM design. There is, therefore, criticism in the use of such correlations, as the geometric domain in which these correlations were tested does not match that of the PJMs. Another example of research of the mixing processes is conducted at NETL's Morgantown site. Testing of the PJM process with non-Newtonian fluid is currently being directed. An experiment investigating the time for a tracer to become fully mixed in the nonNewtonian fluid while undergoing sparging was investigated. There is an interest in understanding the effect of rheological properties and sparging flow rates on the flow characteristics of the PJM vessels.

\subsection{Research Objectives}

The primary focus of this thesis is to conduct Computational Fluid Dynamics (CFD) analysis in order to investigate the criticism arising from applying Poreh's correlation to the PJM design process and to develop a simulation that is capable of studying the effects that rheological properties, characteristic dimensional properties, and sparging flow rates have on the sparging process. An assessment of these two aspects of 
the PJM process is critical to the Successful design and implementation of the PJMs which are used for treatment and storage of nuclear waste. 


\section{CHAPTER II - LITERATURE REVIEW}

Although both research objectives of this thesis address issues of the PJM process, for the sake of organization it is useful to separate the two topics as they are technically different in nature. For this reason each chapter will have a subsection addressing each research objective.

\subsection{Review of Jet Impingement}

The turbulent jets impinging orthogonally on a solid surface have numerous engineering applications that involve heating, cooling and drying operations, and mass transfer in industrial spraying [3]. Similarly, jet impingement is widely seen in environmental engineering processes such as rotorcraft brownout, soil erosion and sediment transport, pollutant discharge in rivers and thunderstorm microburst winds([4],[5]). Another frequently seen practical application of orthogonally impinging jets is in the assessment of mixing performance of two miscible fluids in pulse jet mixing devices in the Waste Treatment and Immobilization Plant [6]. Furthermore, the problem of normal impinging jets is considered to be a standard test case for the development of turbulence models.

Several earlier investigations([7],[2],[8]) have shown that the flow field formed by jet impingement on a solid surface is broadly classified in several distinct regions, such as free jet, impingement and radial wall jet regions, as displayed on the right of Figure 1. Here, $D$ refers to the diameter of the circular jet and $b$ measures the distance from the nozzle to the solid surface. Many factors such as nozzle diameter $(D)$, inflow turbulent condition at jet, and the gap ratio between nozzle to target impinging plate $(b / D)$, affects the jet characteristics as well as the optimum heat transfer augmentation 
process shown by Craft et al. [9]. An extensive review on heat and mass transfer behavior of impinging jets have been reported by Jambunathan et al. [10]. Most of the earlier studies related to impinging jets focused on heat transfer characteristic at the wall. These studies focused on impingement region for $b / D$ in the range of 2.0-10.0 and for low Reynolds number $(20,000)$ applications [3]. Similarly, a number of experimental studies concerning the mean velocity fields have been reported in literature focusing mostly on the stagnation region and nearby wall jet regions, but, again $b / D$ was restricted primarily to being less than 6 , where, the potential core of the jet is in the development region ([8],[11]). Studies on scalar mixing fields or concentration fields involving impinging jets have also been documented by Fairweather et al. [12].

Benchmark data on heat transfer and mean velocity fields in the impingement region or nearby wall jet region of circular impinging jet flows have been reported extensively. So far, very few investigations focus their attention on the self-similar behavior of mean radial velocity profiles in wall jet regions, where the jet characteristics can be described by empirical correlations, as demonstrated by Glauert et al. [13]. The radial wall jet forms upon striking the axial jet on the solid surface and subsequently changes the direction in the impingement region to flow in the radial direction parallel to the wall. The jet characteristics in wall jet regions have practical importance in environmental engineering, such as in mixing of waste at WTP, sediment transport, thunderstorm microburst winds, etc. Glauert was the first to treat the radial wall jet problems theoretically, dividing the flow features in the radial wall jet regions into two separate regions. These regions are composed of an outer free turbulent region and an inner region where the wall effect is dominant. The regions are used to develop 
mathematical relationship of self-similar dimensionless local radial wall jet velocity profiles and growth rate of wall jet boundary layer as a power function of radial distance $r$. Later, Poreh et al. [2], reinvestigated the relationship in more detail through experimental observations of hot-wire measurement. The Experiment entailed a circular air jet impinging normally on a flat surface at a high Reynolds number $\left(\sim 10^{5}\right)$ and established the empirical relationship for local (time averaged) maximum of radial velocity $\left(U_{m}\right)$ and the spreading rate wall jet or length scale $\delta$ referred as jet half-width. $\delta$ was obtained by measuring the vertical distance $z$ at which radial velocity become $0.5 U_{m}$, which is schematically shown in Figure 1. The correlations, which will be referred to as Poreh's correlations in the rest of this thesis, are defined as follows:

Characteristic jet half-width $(\delta), \quad \delta / b=0.098 *(r / b)^{0.9}$

Maximum radial jet velocity $\left(U_{m}\right), \quad U_{m} b / \sqrt{K}=1.32\left(\frac{r}{b}\right)^{-1.1}$

where, $K$ is the kinematic momentum flux of the circular jet at exit, $r$ indicates radial locations along the wall. The measured data was gathered at a large radial distance from the impingement region over $0.75 \leq r / b \leq 2.75$ or $6 \leq r / d \leq 30$, keeping the gap ratio between the nozzle to target impinging plate, $b / d$ more than 8 . The author also mentioned that the wall jet velocity profiles depend on impinging height $b$, even at a large radial distance far away from the stagnation point. A decade later, Beltaos et al. [14] carried out an experimental study of impinging jets at a at low impingement height $(b / d \leq 6)$, and developed empirical correlations to predict the maximum radial velocity $\left(U_{m}\right)$ and spread rate of the jet $(\delta)$ that is valid in the radial stations ranges, $0.75 \leq r / d \leq 3.0$. The authors also found the wall jet region to start at the end of the 
impingement region at $r / b=0.22$ and classified the impinging jet as "small" when, $b / d \leq 5.5$ and "large" for $b / d \geq 8.3$. Similarly, Wood et al. [15] studied the impinging jet with $b / d \leq 3$ for modeling thunderstorm downbursts and proposed an empirical correlation for self-similar radial wall jet velocity profiles which is valid for $0.75 \leq r / d \leq 3.0$. Similarly, Sengupta et al. [5] extensively studied impinging jets for a small impingement height $(b / d \leq 3)$ employing PIV experimental techniques and CFD validation using Large Eddie Simulations (LES) and Reynolds Averaged Navier-Stokes (RANS) models, focusing the radial profiles within the range, $0.75 \leq r / d \leq 3.0$. They proposed new empirical correlations for $U_{m}$, which closely fit Poreh's correlation at large $r / d$, although, the numerical data using various turbulence models was less successful and varied over a small range. More recently, Ghaneeizad et al. [4] conducted an experimental study of impinging jets with a larger impingement height of $b / d=24$, but at a low Reynolds number $(\operatorname{Re}=32,000)$, limiting the radial velocity data within $r / b \leq 1$. Their results showed that the $\delta$ variations slightly diverged from Poreh's correlations. In an another notable experimental study of similar impinging jets, but at a small jet impingement ratio $(b / d=2)$ and at a low Reynolds number $(\operatorname{Re}=20,000)$, Fairwhether et al. [16] reported a slight discrepancy in their measurement data with Poreh's correlation and attributed the differences on the intrusive nature of the single hotwire anemometers employed by Poreh [2].

Review of current literature concerning the self-similar radial wall jet velocity profiles and associated empirical correlations $\left(U_{m}\right.$ and $\left.\delta\right)$, reveals that a majority of the studies employed experimental techniques and limit the $b / D$ ratio to less than 5 . These studies focused the radial locations of extraction close to the impingement region 
in $0.75 \leq r / d \leq 3.0$. However, Poreh's experimental study considers a "high" impingement height $(b / d \geq 8)$ and presents data at radial locations far away from the impingement region, $6 \leq r / d \leq 30$. Interestingly, very few studies tested Poreh's correlation and looked at radial stations very close to the impact region at $r / b \leq 1$. No exhaustive studies were available to compare Poreh's data at large radial distances and for high impingement heights. With regards to numerical simulations, no studies were reported that directly compare Poreh's correlations, except, Sengupta [5] who compared similar impinging jet flows with their own empirical correlations at close radial locations $(r / d \leq 3.0)$ and found the numerical predictions less accurate and data scatter over a small range.

Bearing in mind the above facts, the current study focuses on the last two issues and numerically investigates Poreh's correlations both in "small" and "high" impingement heights and at all radial locations. Especially, the current study investigates jet flows impinging on curved surface considering similar operating conditions as in an actual pulse-jet-mixers (PJM) device. The interest of impinging jet on curved impinging surface stems from its practical application in mixing vessels (PJM) in Waste Treatment Plant at the Hanford site, USA, [6]. The National Energy Technology Laboratory is carrying out a conceptual design and analysis, employing CFD and experimental technique to study mixing performance of PJM devices. The flow features created in PJM mixing devices are very similar to submerged impinging jets where fluid exits and is sucked through nozzles connected to a pulse tube to inject on the bottom curved surface of pressurized vessel. After striking the bottom surface, the flow is redirected to form radial wall jets that collide against each other at the center of the vessel to create an up- 
wash plume which promotes mixing of the waste and the process continues cyclically until desired mixing is achieved. The mechanism of mixing process is shown schematically on the left of Figure 1, in-which, the mixing efficiency depends on the local radial wall jet velocity and spreading rate of jet. In practice, the controlling parameters in PJM vessel design rely on Poreh's correlations[6]. However, the PJM is operated at a "small" impingement height ratio, $b / d \leq 1.5$ with slightly confined conditions and the impingement is not perfectly orthogonal. In-order to account the curvature and confinement issues, Poreh's correlation need to be tested for actual PJM conditions.

\subsection{Review of Bubble Columns}

Bubble columns are widely used in the chemical industry and in several applications within the nuclear energy industry [17]. Specific applications of bubble columns are seen in the production of baker's yeast, citric and amino acids, metals, Microbial fuel cells, and many more [18-22]. These kinds of systems are widely used because they offer favorable mass transfer and mixing characteristics at a feasible cost due to their mechanical simplicity [23].

The existing literature on bubble columns articulates the unsteady behavior flow in a bubble column. This unsteady behavior is a significant factor in the mixing and transport progression in bubble columns flows and occurs at varying spatial and temporal scales [24]. These unsteady features are undoubtedly affected by geometrical factors e.g. liquid height, geometric characteristics, and sparger flow rate. For this reason, experimental works on bubble columns are identified by a liquid height, characteristic length, and a superficial gas velocity range (sparger flow rate). One of the significant 
effects of the mentioned parameters is on the nature of the bubble flow, as shown in Figure 2:

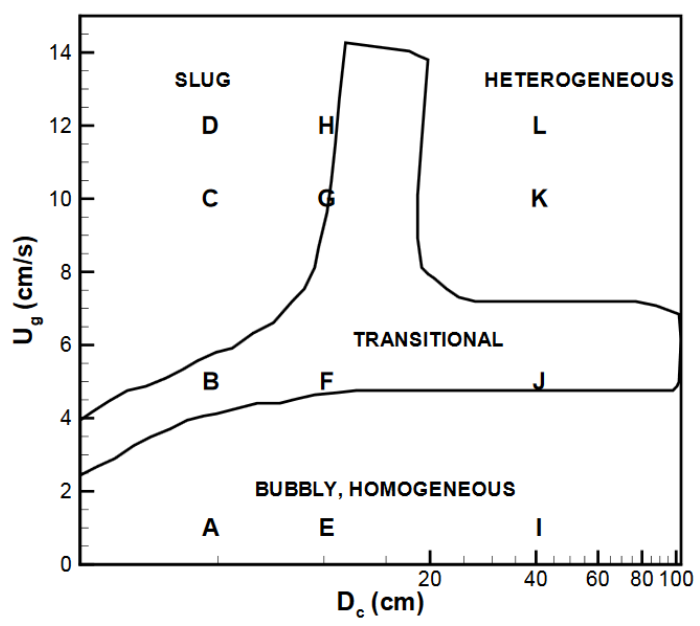

Figure 2-Bubble column flow regime based on characteristic length and flow rate

From Figure 2 it can be discerned that there are four different kinds of bubbly flow in a bubble column as a function of the superficial gas velocity $U_{g}$ and column diameter $D_{c}$. These are homogenous, heterogeneous (Churn-turbulent), transitional, and slug bubbly flow regimes. Homogenous and heterogeneous bubbly flows characterize the distribution of bubble diameters. Slug flows are characterized by agglomeration of bubbles which are observed to be as passing large bubbles. The context of this thesis maintains its focus on homogeneous bubbly flow as that is the flow that is proposed for the PJMs.

In the literature, most of the earlier experimental and computational studies were conducted on rectangular bubble columns and mainly focused on investigating time averaged behavior of a Newtonian fluid. For example Becker et al, Pfleger et al, Pan et al, and Buwa studied rectangular bubble columns [25-29]. According to Rampure et al this was due to the fact that plume oscillations in this geometry were already well established. 
It was also due to simpler geometry, which reduces computational requirements while still retaining key characteristics observed in cylindrical bubble columns.

Experimental studies of cylindrical bubble columns, the shape of focus for this thesis, have also been conducted for Newtonian fluids. For example, Chen et al [30] studied the effect of column size on heat transfer characteristics and volume fraction profile under $\frac{H}{D} \cong$ of $10,5, \& 2.5$ and superficial gas velocities of $.02-.09 \frac{\mathrm{m}}{\mathrm{s}}$. Chen recorded that gas volume fraction profiles were parabolic and symmetric in nature. $\mathrm{He}$ also noted that with increasing $\frac{H}{D}$ there was a flattening of the parabolic gas volume fraction profile. Rampure et al [24] investigated the effects of $\frac{H}{D}=5 \& 10$ under superficial gas velocities of $.01-.2 \frac{\mathrm{m}}{\mathrm{s}}$ on the dynamic and time averaged flow behavior of cylindrical bubble columns. Rampure also noticed that oscillations of bubble swarms had varying time scales, unlike that of rectangular bubble columns. In a later study, Rampure et al [31] also studied bubble columns at higher superficial gas velocities (up to $.4 \frac{\mathrm{m}}{\mathrm{s}}$ ) under a similar characteristic ratio $\left(\frac{H}{D}=5\right)$ by measuring local time-averaged gas holdup and velocity profiles. Rampure also looked into mixing times, where the mixing times asymptotically approached a minimum value as superficial gas velocities were increased. As a more recent example, McClure et al [32] investigated bubble size distribution (BSD) as a function of sparger design using $\frac{H}{D}=2.5$ and superficial gas velocities of .01 to $.11 \frac{\mathrm{m}}{\mathrm{s}}$. McClure observed that BSD in water air systems changed significantly with column height and insignificantly with increasing superficial velocities at the range tested. 
Experimental investigations of cylindrical bubble columns with non-Newtonian fluid are not as plentiful in number as they are with Newtonian fluids. Amin E. et al [33] showed radial distribution of both upward gas velocity and volume fraction for an array of non-Newtonian fluids in a bubble column with a $\frac{H}{D}=2.5$ tested at superficial velocities between .04 and $.2 \frac{\mathrm{m}}{\mathrm{s}}$. Cécile et al [34] similarly studied mixing time in a bubble column using non-Newtonian power law fluids as a function of superficial gas velocities. The experiment had an $\frac{H}{D}=1.75$ and was tested at superficial gas velocities of $.001-.008 \frac{\mathrm{m}}{\mathrm{s}}$. Y. Kawase et al [35] conducted experiments on a bubble column with fermentation broths having a yield stress. In his work he detailed total gas hold up as a function of superficial velocity and axial velocity as a function of superficial velocity for a superficial velocity range of .01 and $.1 \frac{\mathrm{m}}{\mathrm{s}}$. Although this fluid is the one of interest, no information on radial distributions of these quantities is given which would better serve for proper validation. Lastly, Junya Knitta et al [36] investigated cylindrical bubble columns with fluids showing both yield stress and power law behavior. Junya also recorded total gas hold up, but noticed that a certain amount did no escape the bubble column due to the yield stress behavior. This experiment was performed for $\frac{H}{D}=5,10$ under superficial gas velocities of .01 to $.1 \frac{m}{s}$.

In addition to the experimental work conducted on cylindrical bubble columns, CFD simulations in this subject have also been conducted but are limited in number. McClure et al $([32,37])$ have conducted Euler-Euler transient simulations of Newtonian bubble columns, matching axial velocity and volume gas fractions profiles radially at different heights. Mohan et al [31] conducted similar simulations and was able to 
accurately capture data for a range of superficial gas velocities $\left(0-.4 \frac{m}{s}\right)$. Lijia Xu et al [38] also used a Euler-Euler approach to investigate gas volume fraction profiles of a slurry in a bubble column. The viscosity model used in this approach was the Thomas semi-theoretical correlation, which does not take into account yield stress.

The CFD studies mentioned recognized the drag force, lift, particle induced turbulence, and virtual mass force as relevant forces to be considered. Furthermore all three studies used RANS modeling, neglected virtual mass force, and agreed that drag was the most dominant force largely contributing to the dynamics of the problem. Worthy of note is that McClure et al was the only study which took turbulent dispersion into account while Lijia $\mathrm{Xu}$ et al was the only study to incorporate the lift force. All the mentioned CFD studies were conducted using different versions of the commercial code Fluent. Up to the Authors Knowledge, there are no CFD simulation models of bubble columns with power law or visco-plastic fluids.

Given the limited examinations conducted on sparged non-Newtonian fluids it is not surprising that none of the studies investigate the $\frac{\mathrm{H}}{\mathrm{D}}$ and shear thickening effect on non-Newtonian bubble columns. This area of study is beneficial to the intentions of adding sparging as a second means of mixing in the PJM process due to the fact that waste tanks are known to vary in size and thus capacity. These parameters will be the focus of this work. 


\section{CHAPTER III - METHODOLOGY}

\subsection{Radial Wall Jet Impingement}

For the objective to investigate the applicability of Poreh's correlation under PJM geometric and physical conditions, a finite volume method was employed using the commercial code package STAR-CCM+V15 that was independently developed by CDAdapco. All theory is obtained from STAR-CCM+'s online documentation [39] and will be broken down into sections consisting of governing equations, numerical approach, initial conditions, and boundary conditions.

\subsubsection{Governing equations}

The flow being considered is isothermal, single phase, and turbulent. Starting with continuity, the governing equations for single phase turbulent flow will be listed. Below is the continuity equation:

$$
\frac{d}{d t}(\rho)+\nabla \cdot(\rho \vec{v})=S_{m}
$$

Where $\rho$ is the density of the fluid and $\vec{v}$ is its velocity vector. The continuity equation in this case equates to zero because the mass inside the domain stays constant. In addition all time derivatives are zero, as this is a steady state simulation. The momentum equation is then:

$$
\frac{d}{d t}(\rho \vec{v})+\nabla \cdot(\rho \vec{v} \vec{v})=-\nabla P+\nabla \cdot \overline{\bar{\tau}}+\rho \vec{g}+\vec{F}
$$

Where $\mathrm{P}$ is the static pressure, $\overline{\bar{\tau}}$ is the stress tensor, and $\rho \overrightarrow{\mathrm{g}}, \overrightarrow{\mathrm{F}}$ are the gravitational and body force respectively. The stress tensor is defined as follows:

$$
\overline{\bar{\tau}}=\mu_{o}\left(\nabla \vec{v}+\left(\nabla \vec{v}^{T}\right)\right)+\left(\lambda-\frac{2}{3} \mu\right) \nabla \cdot \vec{v} \overline{\bar{I}}
$$


Where $\lambda$ and $\mu$ represent the bulk and shear viscosity, upper script T symbolizes the transpose, and $\overline{\overline{\mathrm{I}}}$ is the identity matrix. Because the flow is incompressible and divergence of velocity is zero, the stress tensor then becomes:

$$
\overline{\bar{\tau}}=\mu_{o}\left(\nabla \vec{v}+\left(\nabla \vec{v}^{T}\right)\right)
$$

\subsubsection{Turbulence}

The Reynolds number is the comparison of inertial forces to viscous forces for a given fluid flow field. It is common knowledge that above a particular Reynolds number for any kind of fluid flow, the flow field transitions from an orderly or laminar flow into a chaotic or turbulent flow. Turbulent flow is characterized by the existence of eddies which have a wide distribution of time and length scales. Furthermore, the larger the Reynolds number the larger the distribution of scales become. In the attempt to model such flows, one must make sure to divide the computational environment into cells which are smaller than the smallest physical phenomena of interest. This method of modeling is called Direct Numerical Simulation. It therefore quickly becomes the case that the computational resources needed to completely resolve all physical phenomena are unfeasible to acquire. One way of resolving this issue is by choosing to ignore a range of smaller length scale eddies, a method referred to as Large Eddie Simulation. Large Eddie Simulations sacrifice accuracy to attain feasibility. Lastly, a common approach in industry to modeling turbulence is to time average the transport equations. This last method is called Reynolds-averaged Navier-Stokes (RANS) and is the method used in all simulations of this thesis. 
For the topic of turbulent round jets, the RANS turbulence model chosen is the standard $k-\varepsilon$. The first such extensive assessment of different turbulence models applied to single impinging jet was performed by Craft el al [9]. They showed less promising results for both the then-widely used standard $k-\varepsilon$ eddy-viscosity model and the basic Reynolds stress model (RSM). They found relatively better success with the non-linear eddy-viscosity model. Later, Behnia et al [40] showed the prediction using Durbin's $v^{2}-f$ model [41] to better agree with the single round jet experiments and provided accurate behavior of heat transfer characteristics. Some more recent studies ([42] [43] ) on single impingement jet resulted in better predictions using the $v^{2}-f$ model and the shear stress transport (SST $k-\omega$ ) turbulence model compared to the $k-\varepsilon$ family [44] leaving an unclear recommendation about appropriate turbulence models.

Summarizing the above discussion reveals that although simple in geometry, the flow in impinging jet system is very complex due to its characteristics, leaving no clear decision about which model is best. The main source of uncertainty in standard $k-\varepsilon$ model arises in the improper behavior of $\varepsilon$ equation in the near-wall layer. The two-layer model (TLM) [45] modifies the inconsistencies of $\varepsilon$ equation in the near-wall layer by specifying improved length scale , thereby, enhance the predictive capability of standard $k-\varepsilon$ model near the wall [46]. Considering the relative performance of various turbulence closures in predicting impinging jet flows, the current work employs standard $k-\varepsilon$ model coupled with two-layer model (TLM) approach. 


\section{Reynolds-averaged Navier-Stokes}

It is not uncommon that in CFD applications the user has no interest on information about the instantaneous flow field. RANS modelling greatly reduces the computational resources needed to conduct a CFD simulation by time averaging the Navier-Stokes equation. This process begins with the idea that all flow variables can be decomposed into a steady mean and a fluctuating part:

$$
V(x, t)=<V(x)>+v^{\prime}(x, t)
$$

Or in more general terms:

Field Variable $(x, t)=<$ Average $>+$ Fluctuations

This idea of separating a field variable by a fluctuating and average part is depicted in the figure below:

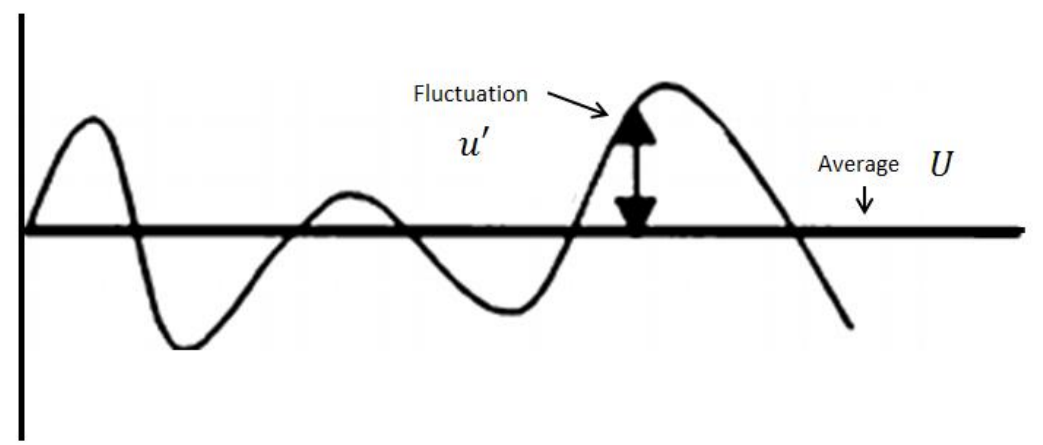

Figure 3- Reynolds decomposition

The time average of a flow variable is defined as:

$$
U(x)=\frac{1}{\Delta t} * \int_{0}^{\Delta t} u^{\prime}(t) d t
$$

Where $\Delta t$ is the time interval in question. By using the definition of time averaging,

Versteeg et al [47] showed that certain rules for time averaging are possible to construct.

Take two decomposed variables A and B:

$$
\begin{aligned}
& A=A(x, t)=\langle A(x)\rangle+a^{\prime}(x, t) \\
& B=B(x, t)=\langle B(x)\rangle+b^{\prime}(x, t)
\end{aligned}
$$

Then: 


$$
\begin{array}{cc}
<\frac{\boldsymbol{d} \boldsymbol{A}}{\boldsymbol{d} \boldsymbol{x}}>=\frac{\boldsymbol{d}<\boldsymbol{A}>}{\boldsymbol{d} \boldsymbol{x}} & <\boldsymbol{A}+\boldsymbol{B}>=<\boldsymbol{A}>+<\boldsymbol{B}> \\
<\boldsymbol{A} * \boldsymbol{B}>=<\boldsymbol{A}><\boldsymbol{B}>+<\boldsymbol{a}^{\prime} * \boldsymbol{b}^{\prime}>\quad & <a^{\prime}>=0 \\
<\boldsymbol{a}^{\prime} * \boldsymbol{b}^{\prime}>\neq 0 & <>* B>=<A><B>
\end{array}
$$

\section{Table 1- Time Averaging Rules}

Applying these rules to the continuity and Navier stokes equations and expressing them in vector notation yields:

$$
\begin{gathered}
\frac{\delta}{\delta t}\left(\alpha_{o} \rho_{o}\right)+\frac{\delta}{\delta x_{i}}\left(\alpha_{o} \rho_{o} \overrightarrow{V_{o, l}}\right)=0 \\
\frac{\delta}{\delta t}\left(\rho_{o} \overrightarrow{V_{o, l}}\right)+\frac{\delta}{\delta x_{i}}\left(\rho_{o} \overrightarrow{V_{o, l} \overrightarrow{V_{o, j}}}\right)=-\frac{\delta p}{\delta x_{i}}+\frac{\delta \tau_{o, i, j}}{\delta x_{i}}+\frac{\delta \tau_{R e, o, i, j}}{\delta x_{j}}
\end{gathered}
$$

Where $V$ is average velocity and $\tau_{o, i, j}$ is the Reynolds averaged stress tensor for phase o:

$$
\tau_{o, i, j}=\mu\left(\frac{\delta V_{o, i}}{\delta x_{j}}+\frac{\delta V_{o, j}}{\delta x_{i}}+\frac{2}{3} \delta_{i j} \frac{V_{o, l}}{x_{l}}\right)
$$

From time averaging the new term $\tau_{R e, o, i, j}$ referred to as Reynolds stress tensor arises:

$$
\tau_{R e, o, i, j}=-\rho \overline{v_{\imath}^{\prime} v_{J}^{\prime}}
$$

This new term arises when time averaging the non-linear convective term. By observing the averaging rules in Table 1 it is discerned that the product of two fluctuating quantities is not zero. The Reynolds stress tensor represents the effect of velocity fluctuations on the mean flow due to increased eddy formation. This term must then be modeled in order to close the system of equations. 


\section{RANS Closure Models}

Standard $\boldsymbol{k}-\boldsymbol{\varepsilon}$

The standard $k-\varepsilon$ was developed by Launder and Spalding (1973) and proposed the following:

$$
\mu_{t}=C_{v_{t} l_{t}} \rho v_{t} l_{t}
$$

Where the subscript $t$ is a reference to the word turbulent, and $l_{t}$ is the turbulent length scale. $l$ can be defined as a function of turbulent kinetic energy $k$ and dissipation of energy $\varepsilon$ :

$$
l=\frac{C_{l} k^{\frac{3}{2}}}{\varepsilon}
$$

And $v_{t}$ can be expressed as a function of turbulent kinetic energy:

$$
v^{2}=k
$$

Plugging back the turbulent length and velocity scales as a function of $k$ and $\varepsilon$ yields the following turbulent viscosity:

$$
\mu_{t}=\rho C_{\mu t} \frac{k^{2}}{\varepsilon}
$$

Where:

$$
C_{\mu t}=C_{l} * C_{v_{t} l_{t}}
$$

Furthermore, Launder and Spalding (1973) suggested the following values for constants based on experimental data:

\begin{tabular}{|ccccc|}
\hline $\boldsymbol{C}_{\boldsymbol{\mu}}$ & $\boldsymbol{C}_{\mathbf{1 , \epsilon}}$ & $\boldsymbol{C}_{2, \boldsymbol{\epsilon}}$ & $\boldsymbol{\sigma}_{\boldsymbol{k}}$ & $\boldsymbol{\sigma}_{\boldsymbol{\varepsilon}}$ \\
\hline .09 & 1.44 & 1.92 & 1 & 1.3 \\
\hline
\end{tabular}

Table 2- Standard $k$ - $\varepsilon$ Model Constants 
Given the assumptions made by Launder et al (1973), the transport equation for turbulent kinetic energy and dissipation become:

$$
\begin{gathered}
\frac{\delta}{\delta x_{j}}\left(\left(\mu+\frac{\mu}{\sigma_{k}}\right) \frac{\delta k}{\delta x_{j}}\right)+\left(G_{k}-\rho \varepsilon\right)+\rho \Pi_{k} \\
\frac{\delta}{\delta x_{i}}\left(\rho \varepsilon V_{i}\right)=\frac{\delta}{\delta x_{j}}\left(\left(\mu+\frac{\mu_{t}}{\sigma_{\epsilon}}\right) \frac{\delta \varepsilon}{\delta x_{j}}\right)+* \frac{\varepsilon}{k}\left(C_{1, \varepsilon} * G_{k}-C_{2, \varepsilon} \rho \varepsilon\right)+\rho \Pi_{\varepsilon}
\end{gathered}
$$

Where $G_{k}$ is a source term for the production of turbulent kinetic energy:

$$
G_{k}=\mu_{k} S^{2}
$$

And $\mathrm{S}$ is the modulus of the mean strain rate tensor:

$$
S=\sqrt{S_{i j} S_{i j}}
$$

Turbulent two way coupling is accounted by the symbols $\Pi_{k} \& \Pi_{\varepsilon}$. The constants $C_{\mu}, C_{1, \epsilon}, C_{2, \epsilon}, \sigma_{k}, \sigma_{\varepsilon}$ are the ones defined in Table 2.

The underlying assumption in the standard $k-\varepsilon$ model is that the flow is completely turbulent, as stated by Launder et al [48]. This assumption is observed in the turbulent dissipation term, where the rate at which energy is transferred from larger to smaller eddies is approximated by the rate of energy that the large eddies extract from the flow in question.

\subsubsection{Numerical Approach}

In STAR-CCM+, integral equations are used to solve the integral conservations of mass and momentum in a sequential manner. The non-linear governing equations are solved iteratively one after the other for the solution variables. 


\subsubsection{Solver algorithm}

The segregated flow solver is used in this work. The segregated solver employs a pressure-velocity coupling algorithm where the mass conservation constraint on the velocity field is fulfilled by solving a pressure-correction equation. The pressurecorrection equation is constructed from the continuity equation and the momentum equations such that a predicted velocity field is sought that fulfills the continuity equation, which is achieved by correcting the pressure.

The pressure-velocity coupling semi implicit algorithm used is called SIMPLE. The algorithm in STAR-CCM+'s theory manual is summarized as follows:

1. Set the boundary conditions.

2. Computed the reconstruction gradients of velocity and pressure.

3. Compute the velocity and pressure gradients.

4. Solve the discretized momentum equation. This creates the intermediate velocity field $v$.

5. Compute the uncorrected mass fluxes at faces $\dot{m}_{f}$.

6. Solve the pressure correction equation. This produces cell values for the pressure correction $\mathrm{p}^{\prime}$

7. Update the pressure field:

$$
\mathrm{p}^{\mathrm{n}+1}=p^{n}+\omega p^{\prime}
$$

Where $\omega$ is the under-relaxation factor for pressure.

8. Update the boundary pressure corrections $p_{b}^{\prime}$.

9. Correct the face mass fluxes:

$$
\dot{m}_{f}^{\mathrm{n}+1}=\dot{m}_{f}+\dot{m}_{f}{ }^{\prime}
$$


10. Correct the cell velocities:

$$
V_{p}^{\mathrm{n}+1}=V_{p}-\frac{v \nabla p^{\prime}}{a_{\prime^{\prime}}}
$$

Where $\nabla p^{\prime}$ the gradient of the pressure corrections is, $a_{p}{ }^{\prime v}$ is the vector of central coefficients for the discretized velocity equation, and $V$ is the cell volume.

11. Update density due to pressure changes.

12. Free all temporary storage.

\subsubsection{Discretization Schemes}

\section{Momentum and Turbulence}

The momentum and turbulence equations are discretized using second order upwind method. For a single direction, the second order upwind scheme uses information at three points:

$$
u_{x}=\frac{3 u_{i}^{n}-4 u_{i-1}^{n}+u_{i-2}^{n}}{2 \Delta x}
$$

Where $u_{i}$ is the value at the current cell or vertex and $\Delta x$ is the distance between the two points.

\section{Gradients}

Gradients are computed using the Green-Gauss gradient method. This method is used for unstructured grids, which uses the green-gauss theorem. This theorem states that the surface integral of a scalar function is equal to the volume integral of the gradient of the scalar function.

$$
\int_{V}^{\cdot} \nabla \phi d V=\int_{V}^{\cdot} \phi d a
$$

This can be written in discrete form to compute the gradient:

$$
\nabla \phi_{r}^{u}=\frac{1}{v_{o}} \sum_{f} \phi_{f} a_{f}
$$


Where the face value is approximated by the arithmetic average of the adjacent cell values:

$$
\phi_{f}=\frac{\phi_{1}+\phi_{2}}{2}
$$

\subsubsection{Rheological modeling}

\section{Newtonian Fluids}

Sir Isaac Newton described the flow behavior of fluids with a linear relationship between shear stress and shear rate, which is why they are referred to as Newtonian fluids. This viscosity model has the shear stress related to the shear rate by a constant, referred to as viscosity:

$$
\tau=\eta * \dot{\gamma}
$$

Where $\tau(p a), \eta(P a-s)$, and $\gamma\left(\frac{1}{s}\right)$ are the shear stress, viscosity, and shear rate respectively. Typical Newtonian fluids are water, honey, milk, air, etc. The viscosity constant is typically calculated by the slope of the line in a shear stress-shear rate curve of the fluid in question. This of course is assuming an isothermal environment, for viscosity is sensitive to temperature gradients.

\subsection{Sparging of Non-Newtonian Fluids in Bubble Columns}

For the objective to investigate rheological and physical effects on a sparged nonNewtonian fluid a finite volume method was employed using the commercial code ANSYS Fluent v16 All the theory is obtained from the Ansys fluent theory manual [49] and will be broken down into sections consisting of governing equations, numerical approach, initial conditions, and boundary conditions. 


\subsubsection{Governing equations}

The Eulerian-Eulerian multiphase flow model in ANSYS Fluent V14.5 is chosen to simulate all bubble column simulations. An alternative to the Eulerian-Eulerian model for bubbly two phase flow is the Eulerian-Lagrangian model, where bubbles are individually tracked as a discrete phase. The Eulerian-Lagrangian model for this reason becomes computationally intensive for large quantities of discrete phases, leaving the Eulerian-Eulerian model the feasible alternative for this work.

The main characteristic of the Eulerian-Eulerian model is that it mathematically represents each phase as interpenetrating continua. In addition, the volume of a phase cannot be occupied by another phase, introducing the concept of volume fraction. The sum of all volume fractions is equal to one for all space and time. This is mathematically expressed in ANSYS Fluent V14.5 user's manual for each phase as:

$$
\sum_{o=1}^{n} \alpha_{o}=1
$$

Where $\alpha$ represents the volume fraction of phase o. In the bubble column under question only two phases exist, namely the non-Newtonian liquid and gas in the form of air. The liquid is referred to as the primary phase and the gas is referred to as the secondary phase. The effective density of each phase is calculated in the following manner:

$$
\widetilde{\rho_{o}}=\alpha_{o} \rho_{o}
$$

Where $\rho_{0}$ is the density of phase o. Furthermore the conservation of mass and momentum laws are fulfilled by each phase, meaning that a set of equations is solved per phase. The continuity equation for phase $o$ is:

$$
\frac{d}{d t}\left(\alpha_{o} \rho_{o}\right)+\nabla \cdot\left(\alpha_{o} \rho_{o} \overrightarrow{v_{o}}\right)=\sum_{p=1}^{n}\left(m_{p o}-m_{o p}\right)
$$


Where $\overrightarrow{v_{o}}$ is the velocity vector of phase o and $\mathrm{p}$ represents the second phase. In this study no mass transfer or reactions occur, equating the continuity equation to zero.

The conservation of momentum equation for each phase o is:

$$
\begin{gathered}
\frac{d}{d t}\left(\alpha_{o} \rho_{o} \overrightarrow{v_{o}}\right)+\nabla \cdot\left(\alpha_{o} \rho_{o} \overrightarrow{v_{o}} \overrightarrow{v_{o}}\right)= \\
\ldots+\left(\overrightarrow{F_{o}}+\overrightarrow{F_{v m, o}}\right)
\end{gathered}
$$

Where $P$ is the pressure, $\overline{\overline{\tau_{o}}}$ is the stress tensor, $\vec{g}$ is gravity, $K_{p o}$ represents the interphase momentum exchange coefficient, $\overrightarrow{F_{o}}$ is the body force, and $\overrightarrow{F_{v m, o}}$ is the virtual mass force.

The first term on the left hand side of equation 6 represents the transient changes in momentum and the second term is the changes of the convection term. The first term on the right hand side takes into account pressure changes. The second term on the right hand side represents the stress tensor term, defined by:

$$
\overline{\overline{\tau_{o}}}=\alpha_{o} \mu_{o}\left(\nabla \overrightarrow{v_{o}}+\left(\nabla{\overrightarrow{v_{o}}}^{T}\right)\right)+\alpha_{o}\left(\lambda_{o}-\frac{2}{3} \mu_{o}\right) \nabla \cdot \overrightarrow{v_{o}} \overline{\bar{I}}
$$

Where $\lambda_{o}$ and $\mu_{o}$ represent the bulk and shear viscosity of phase o, upper script T symbolizes the transpose, and $\overline{\bar{I}}$ is the identity matrix. Furthermore, the third term takes into account acceleration due to gravity. The fourth term includes the interaction forces between the two phases as well as mass transfer between phases. The fifth and final term on the right hand side represents the body force and the virtual mass forces.

In the context of this work there is no mass transfer and the compressibility of phases will be neglected, yielding the following conservation of mass equation:

$$
\frac{d}{d t}\left(\alpha_{o} \rho_{o}\right)+\nabla \cdot\left(\alpha_{o} \rho_{o} \overrightarrow{v_{o}}\right)=0
$$


And the following stress tensor:

$$
\overline{\overline{\tau_{o}}}=\alpha_{o} \mu_{o}\left(\nabla \overrightarrow{v_{o}}+\left(\nabla{\overrightarrow{v_{o}}}^{T}\right)\right)
$$

The interphase exchange coefficient is a function of the drag. The Schiller-Naumann drag model is used as it is well accepted in the area of bubbly multiphase flows ([17], [50]). The drag force predicted by Schiller-Naumann model is described as follows:

$$
f=\frac{c_{D} R_{e}}{24}
$$

Where $c_{D}$ the coefficient of drag and $R_{e}$ is the Reynolds number. The Coefficient of drag is computed in the following manner:

$$
c_{D}=\left\{\begin{array}{lr}
\frac{24\left(1+.15 R_{e} e^{.687}\right)}{R_{e}}, & R_{e} \leq 1000 \\
.44, & R_{e} \geq 1000
\end{array}\right.
$$

Where:

$$
R_{e}=\frac{\rho_{o}\left|\overrightarrow{v_{p}}-\overrightarrow{v_{o}}\right| d_{b}}{\mu_{o}}
$$

\subsubsection{Turbulence}

The turbulence model chosen for this multiphase flow is the RANS $\boldsymbol{k}-\boldsymbol{\varepsilon}$ RNG model. Since the fundamental assumptions and equations have already been underlined previously in the jet impingement section, the RANS closure model will only be included in this section. It is important to note that the standard $k-\varepsilon$ model was originally intended for single phase flow but several studies such as the ones conducted by Dale McClure ,Rampure et al, Sokolichin, and Eigenberger [24, 32, 51] have shown that the standard $k$ $\varepsilon$ models give physically accurate results for bubble columns. This fact supports the use

of the $\boldsymbol{k}-\boldsymbol{\varepsilon}$ RANS models in bubble column simulations. Furthermore according to Xe et al [52], Laborde-Boutet et al. [53] investigated the implementation of different $k-\varepsilon$ models and compared simulation results with experimental data of Chen [54]. The results 
indicated that RNG $k-\varepsilon$ model was best able to capture the flow characteristics in bubble columns within the turbulent regime.

\subsubsection{RANS Closure Model}

$k-\varepsilon R N G$ (Renormalization Group)

This RANS model addresses the assumption that standard $k-\varepsilon$ is only applicable to fully turbulent flows. Yakhot et al [28] developed the RNG model so that the model constants are not experimentally obtained, but rather calculated theoretically using a statistical technique known as Renormalization Group. The constants developed are listed below:

\begin{tabular}{|c|c|c|c|c|c|}
\hline$C_{\mu}$ & $C_{1, \epsilon}$ & $C_{2, \epsilon}$ & $\sigma_{k}$ & $\sigma_{\varepsilon}$ & $\eta_{0}$ \\
\hline .0845 & 1.42 & 1.68 & .7194 & .7194 & 4.38 \\
\hline
\end{tabular}

Table 3-Model Constants for $k-\varepsilon$ RNG Turbulence Model

A differential formulation of the eddy viscosity which accounts for low Reynolds number effect is also included.

\subsubsection{Numerical Approach}

In Fluent, integral equations are used to solve the conservation of mass and momentum equations. These equations are not yet solvable analytically, so it is necessary to use numerical techniques to discretize them.

\subsubsection{Solver algorithm}

The pressure based solver is used in this work. It leverages concepts of continuity and conservation of momentum to obtain the velocity field, which is then corrected by the pressure field and must always satisfy mass conservation. This process is repeated until a stable or converged solution is reached. 
The pressure based solver solves the continuity and momentum equations separately. The phase-coupled semi-implicit (PC-SIMPLE) algorithm is chosen for this task. As the name implies, through the coupling of phases the velocities are solved and a block algebraic multigrid scheme is employed to solve the vector equations. The pressure $P$ and velocity $u$ are obtained in the following manner:

$$
\begin{aligned}
& P=P_{o}+\beta_{p} P^{\prime} \\
& u=u_{o}+u^{\prime}
\end{aligned}
$$

Where the subscript o indicates that that variable is an initially guessed value $\beta_{p}$ is a relaxation factor for pressure, and supper script ' indicates that the variable is corrected. The corrected pressure and velocity, $P^{\prime}$ and ' , are approximately related by the following:

$$
u^{\prime}=-A \frac{\delta P^{\prime}}{\delta x}
$$

Where A is an arbitrary time increment divided by density. The velocity estimation and velocity correction equations are combined to obtain the pressure correction term:

$$
A \frac{\delta^{2} P^{\prime}}{\delta x^{2}}-\frac{\delta u_{o}}{\delta x}=0
$$

In this manner, the pressure and velocity are iteratively guessed and corrected until the corrected and guessed terms converge to the same value, within a desired error.

\subsubsection{Discretization Schemes}

\section{Volume Fraction}

The quadratic upwind interpolation for convective kinematics (QUICK) is applied to volume fraction discretization. These kinds of schemes are based on a weighted average of second order upwind and central interpolations of any variable, $\phi$, in 
question. Figure 4, taken from the fluent theory guide, shows how $\phi$ can be written for face e assuming the flow direction is from left to right:

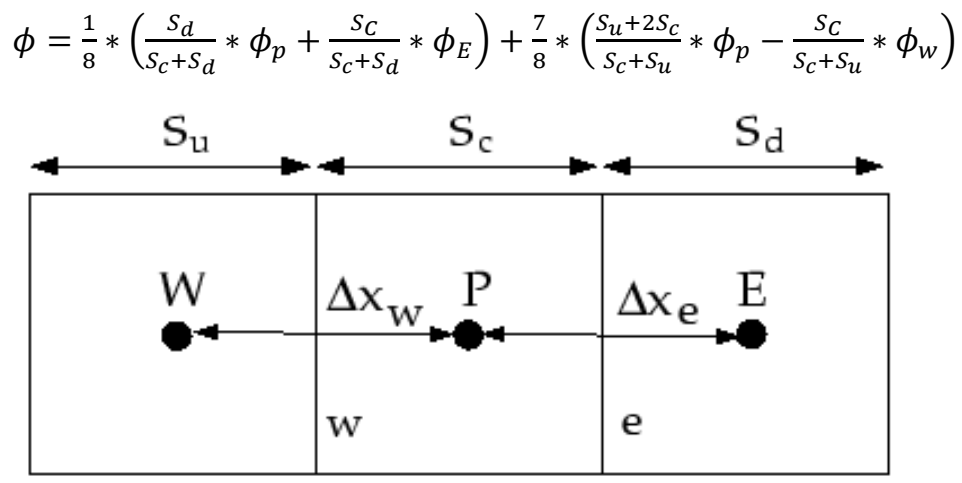

Figure 4- 1 Dimensional control volume

We see from Figure 4 that each cell has a size $S$ and variable value $\phi$ stored at the cell center.

\section{Momentum and Turbulence}

In order to discretize the momentum and turbulence equations the third order MUSCL (Monotone Upstream-Centered Schemes for Conservation Laws) was used. This scheme was created by blending a central differencing scheme and a second-order upwind scheme as:

$$
\phi_{f}=\theta \phi_{f, C D}+(1-\theta) * \phi_{f, S O U}
$$

Where $\theta \phi_{f, C D}$ is the central differencing of the variable $\phi$ and $\phi_{f, S O U}$ is the second order central differencing of the same variable.

\section{Gradients}

To evaluate the gradients of a variable, the Least Squares Cell-Based method was used. This method assumes the solution to vary linearly. The change in cell values between cell $\mathrm{Co}$ and $\mathrm{Ci}$ along the vector $\mathrm{r}_{\mathrm{j}}$ from the centroid of cell $\mathrm{Co}$ to $\mathrm{Ci}$, as seen in Figure 5 , can be written as: 


$$
\nabla \phi_{c o} \cdot \nabla r_{i}=\phi_{c i}-\phi_{c o}
$$

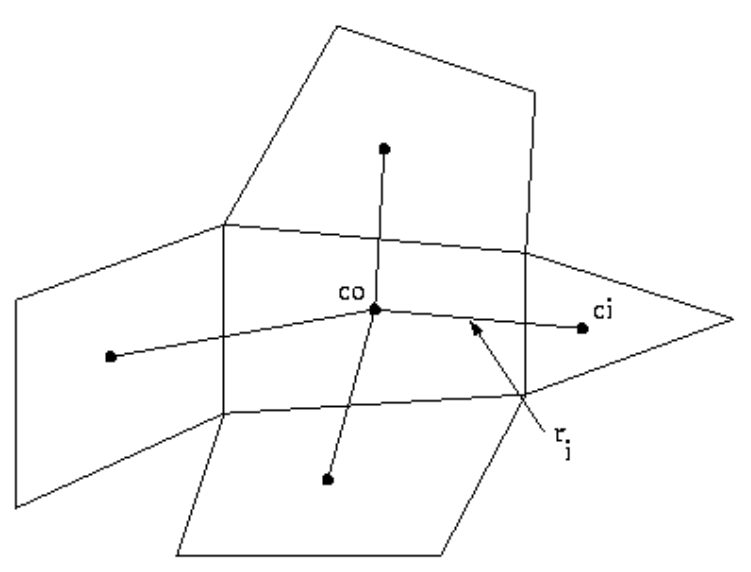

Figure 5-Cell centroid

This equation can be written for each cell around Co to obtain a system of equations:

$$
[J] * \nabla \phi_{c o}=\Delta \phi
$$

Where the $J$ matrix described the geometry or $r_{j}$ vector for each surrounding cell. This system of equations is then solved by decomposing the J matrix using the Gram-Schmidt process. Further details of this process can be obtained in the fluent theory guide [49].

\section{Temporal}

Lastly, the time derivative was discretized using a first order implicit method available in fluent. Considering the one-dimensional transient differential equation below:

$$
\frac{\delta \varphi}{\delta t}=y(\varphi)
$$

Where $y(\varphi)$ is a general function of $\varphi$. If the above equation is integrated over one time step $\Delta t$ it results in the following:

$$
\frac{\varphi^{n+1}-\varphi^{n}}{\Delta t}=y(\varphi)
$$


where $\mathrm{n}$ represents the current time level. Evaluating $y(\varphi)$ at the future time level, $\mathrm{n}+1$, is what denotes this method as implicit. Rearranging the terms to solve for the next time step yields:

$$
\varphi^{n+1}=\varphi^{n} \Delta t y\left(\varphi^{n+1}\right)
$$

\subsubsection{Rheological modeling}

\section{Non-Newtonian Fluids}

In fluent, there are a number of ways to model non-Newtonian fluids. For the scope of this work the power law model in fluent is discussed. The non-Newtonian viscosity for isothermal conditions is given by:

$$
\eta=k * \dot{\gamma}^{n-1}
$$

where $\mathrm{k}$ and $\mathrm{n}$ are the consistency index and power law index respectively. The value of the power law index determines what kind of fluid it is. If $\mathrm{n}$ is equal to one then it is a Newtonian fluid. If $\mathrm{n}$ is greater than one it is referred to as a shear thickening fluid and lastly, if $\mathrm{n}$ is less than one the fluid is shear thinning. 


\section{CHAPTER IV - Numerical Investigations}

\subsection{Radial Wall Jet impingement}

The main focus of this sections work is to investigate the applicability of Poreh's correlations on the PJM geometric and physical conditions. This is achieved by first simulating Poreh's original experiment and matching the experimental data. Then that same simulation is altered geometrically to have the PJM's aspect ratio $(b / D)$. Finally this simulation is then geometrically altered once more to reflect the curvature of the waste tanks during the PJM process.

\subsubsection{Replication of Poreh's Experiment and Reduction of Aspect Ratio (b/D)}

In Poreh's experimental study, air jet was issued at velocity of $U_{0}=340 \mathrm{ft} / \mathrm{s}$

from a circular orifice of diameter $d=2 \mathrm{in}$. located at a fixed normal distance of $b=2 \mathrm{ft}$ above a circular flat plate with a radius of 69 inches. The isothermal air jet at room temperature from the orifice impinges normally on the bottom flat surface and subsequently turns around to spread radially outwards on the flat surface to form symmetrical radial jets around the impingement point. The Reynolds number based on kinematic momentum flux $(K)$ or the jet exit velocity was $\operatorname{Re}=1.96 \times 10^{5}$. The wall normal distribution of radial wall jet velocity profiles at various radial locations far away from the impingement region were measured and the maximum radial wall jet velocity $\left(U_{m}\right)$ and jet-half width $(\delta)$ were reported as standard correlations. The schematic on the right of Figure 1 depicts the physical environment in Poreh's experiment. Figure 6 below depicts the simulated environment developed in STAR$\mathrm{CCM}+$ for both Poreh's experiment and the altered simulation reflecting the PJM characteristic ratio of 1.5 . 


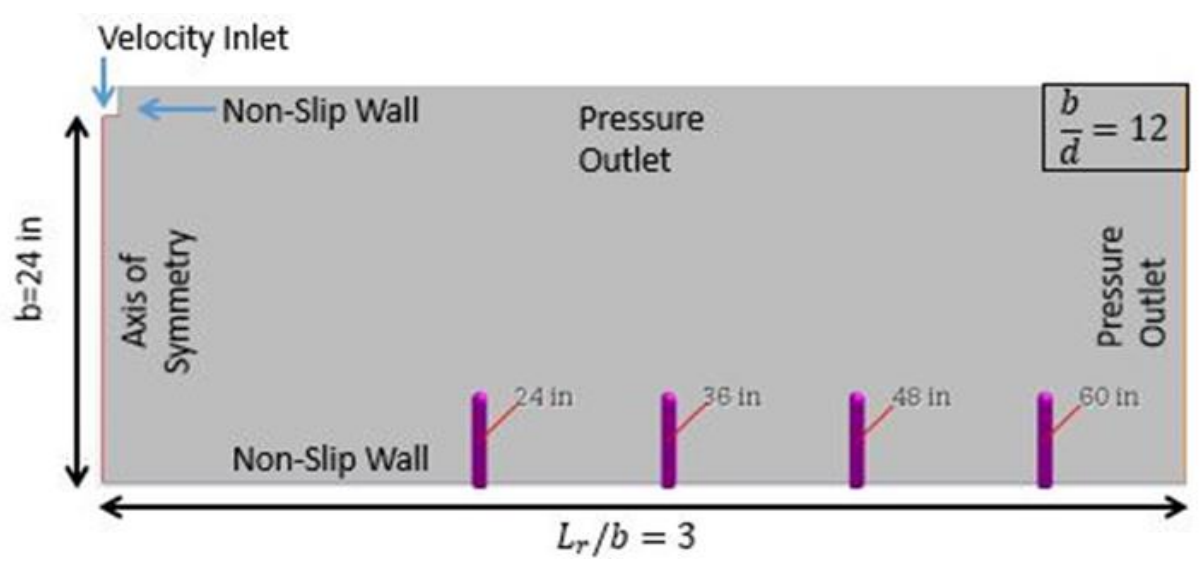

(a) Domain with $b / d=12$

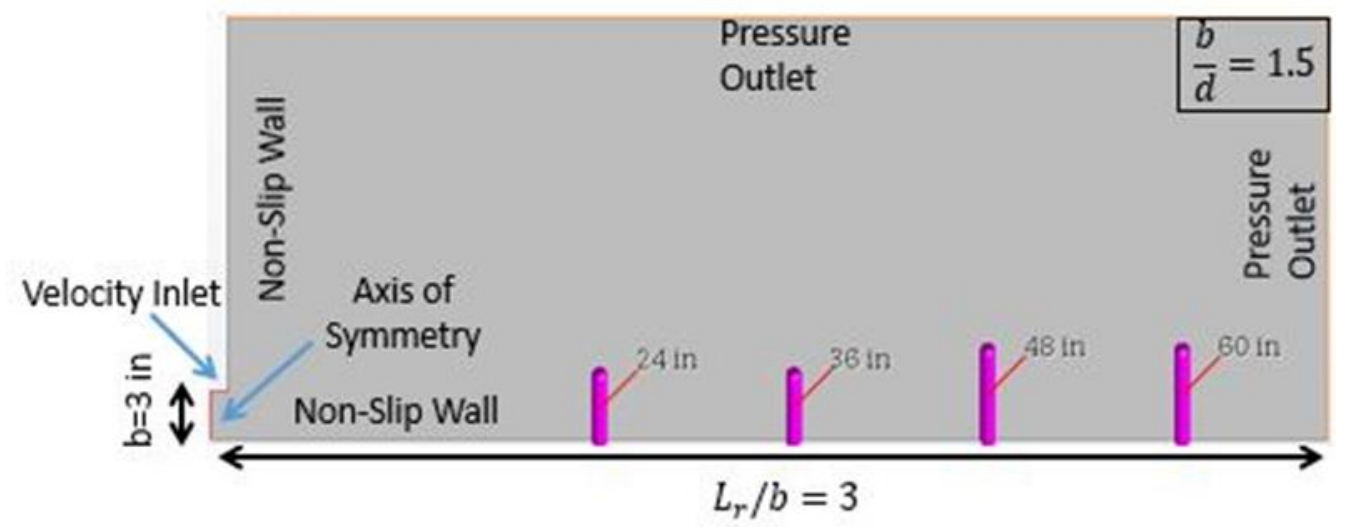

(b) Domain with $b / d=1.5$

Figure 6- Schematic of computational domain showing geometrical dimensions, boundary conditions, and data extraction lines for (a) Poreh's experiment (b) and altered simulation reflecting lower b/d ratio

As can be observed from Figure 6, an axis-symmetric boundary condition was chosen to model Poreh's experiment. This decision was based on that Poreh's experimental data varied insignificantly in the circumferential direction. This allows for the assumption of an axi-symmetric boundary condition to be a valid simplification. The simulation in Figure 6 (a) is tested for robustness through a grid sensitivity analysis in Figure (7): 


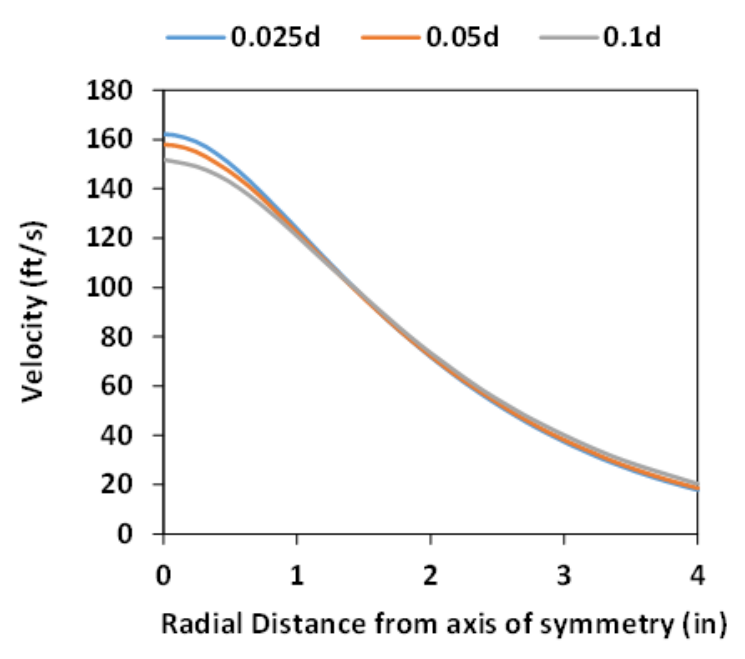

(a) Circular Jet region

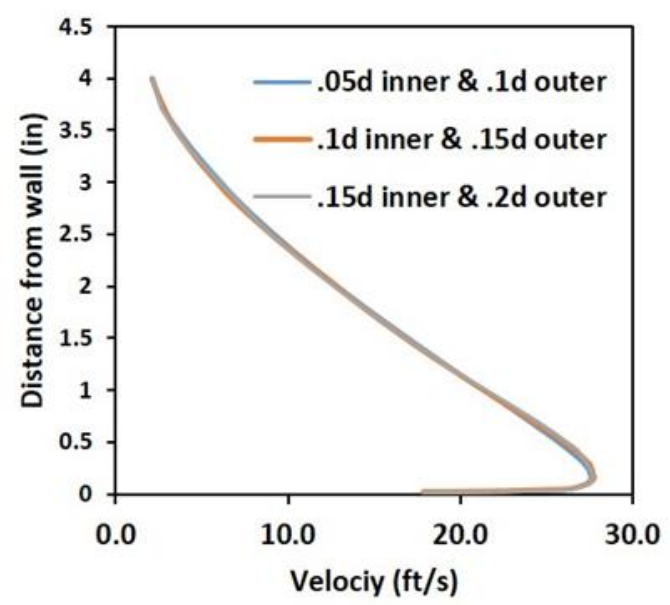

(b) Radial wall jet region at $r / b=1$

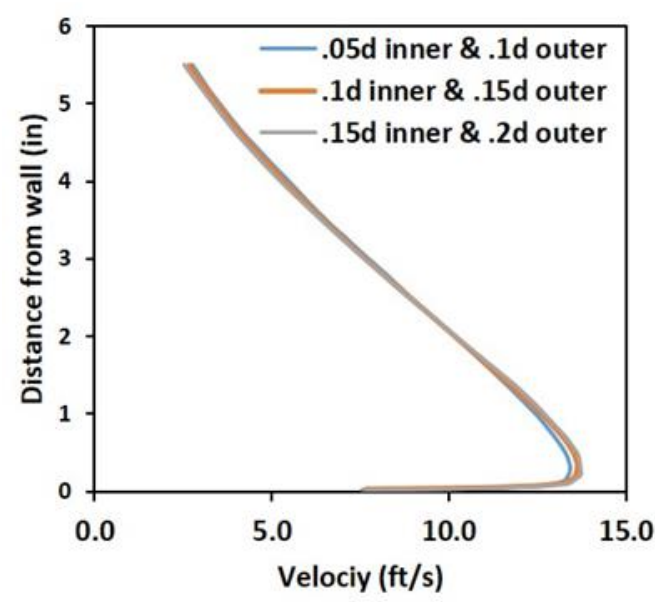

(c) Radial wall jet region at $r / b=2$

Figure 7- Grid sensitivity test on mean velocity profiles (a) Circular Jet region (b) Radial wall jet region at $r / b=1$ and (c) Radial wall jet region at $r / b=2$

In Figure 7 one can see that as the mesh is refined the results do not change neither in the round jet region (Figure 7a) nor radially outward at two distinct locations. The characteristic features of a radial wall jet velocity profile can also be observed in Figure 7 $\mathrm{b}$ and $\mathrm{c}$. The final meshes for both simulations are depicted below: 


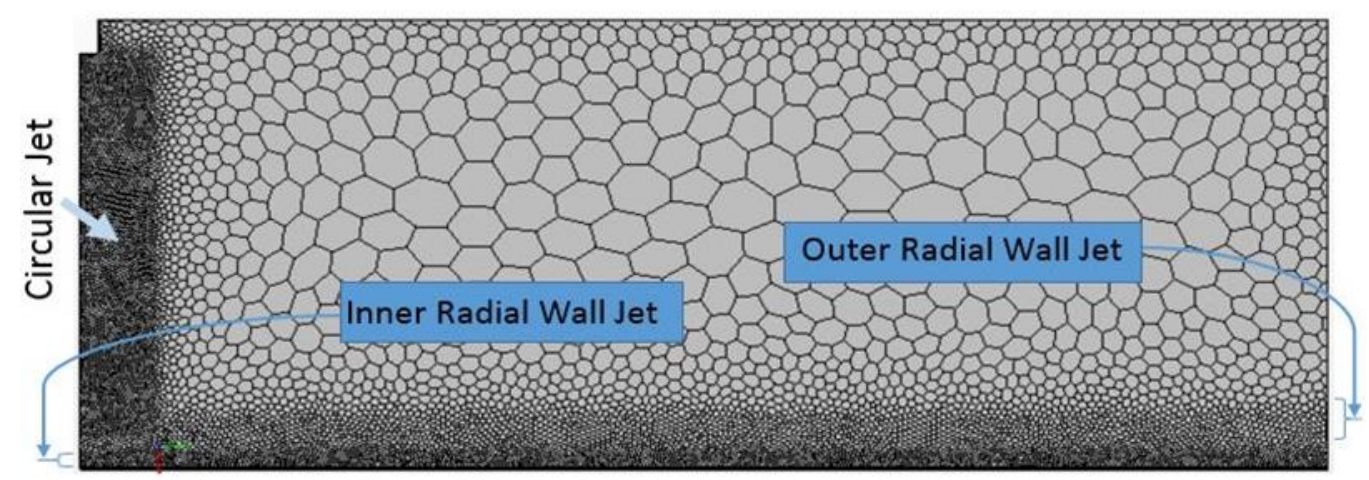

(a) Computational mesh of flat surface geometry with $b / d=12$

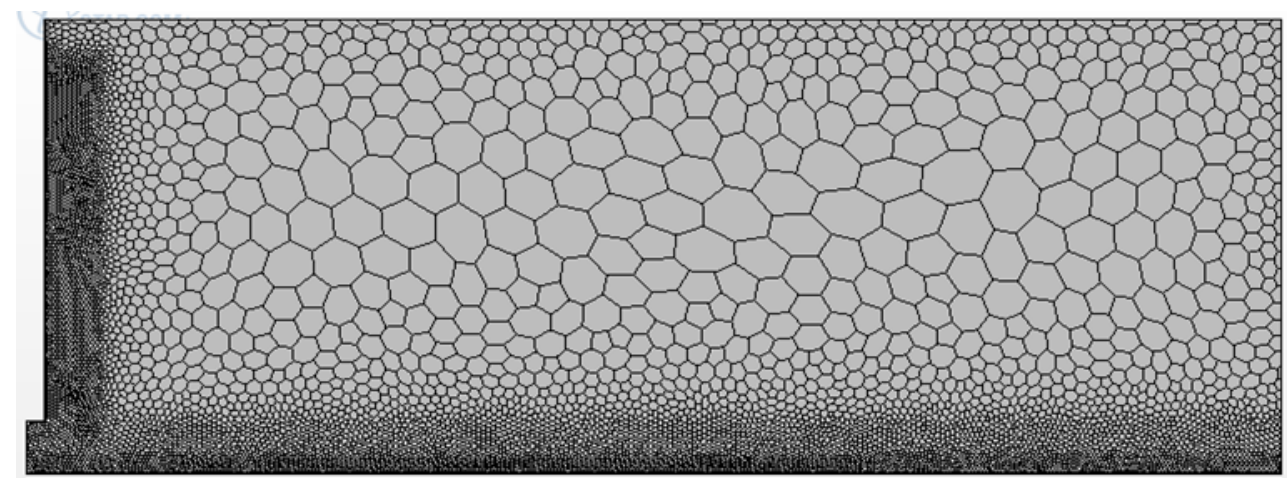

(b) Computational mesh of flat surface geometry with $b / d=1.5$

Figure 8-Computational mesh showing polyhedral mesh properties $(a) b / d=12(b) b / d=1.5$

It can be observed In Figure 8 that mesh refinement regions were implemented in areas

where large velocity gradients are expected, namely the circular jet and the radial wall jet.

The radial wall jet region was separated into an inner radial wall jet and an outer radial wall jet, where velocity gradients are expected to decrease with increasing distance from the impingement surface. Coarsening of the mesh is implemented where stagnant or relatively low velocity gradients are expected. Once mesh independency was established both simulations in Figure 6 were ran. The results are summarized below: 


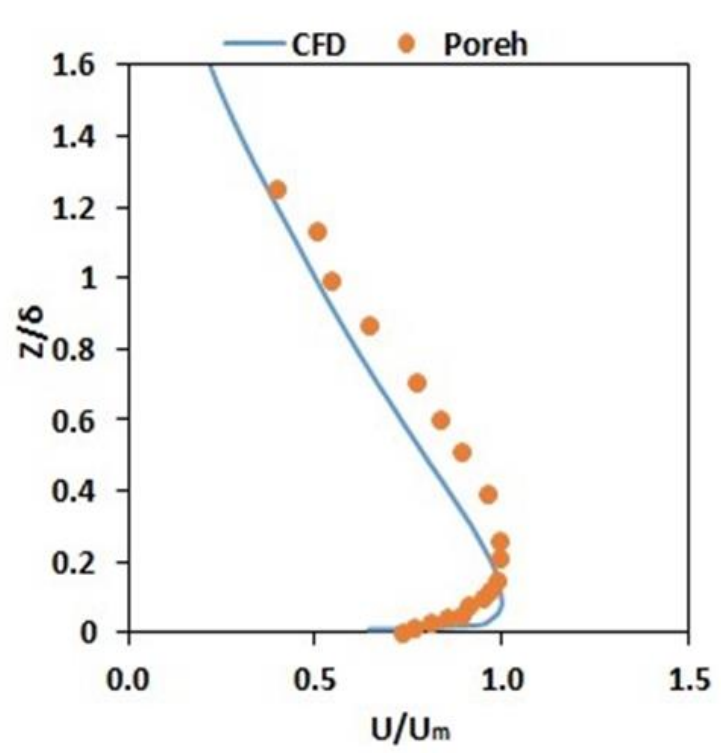

(a) $r / b=1$

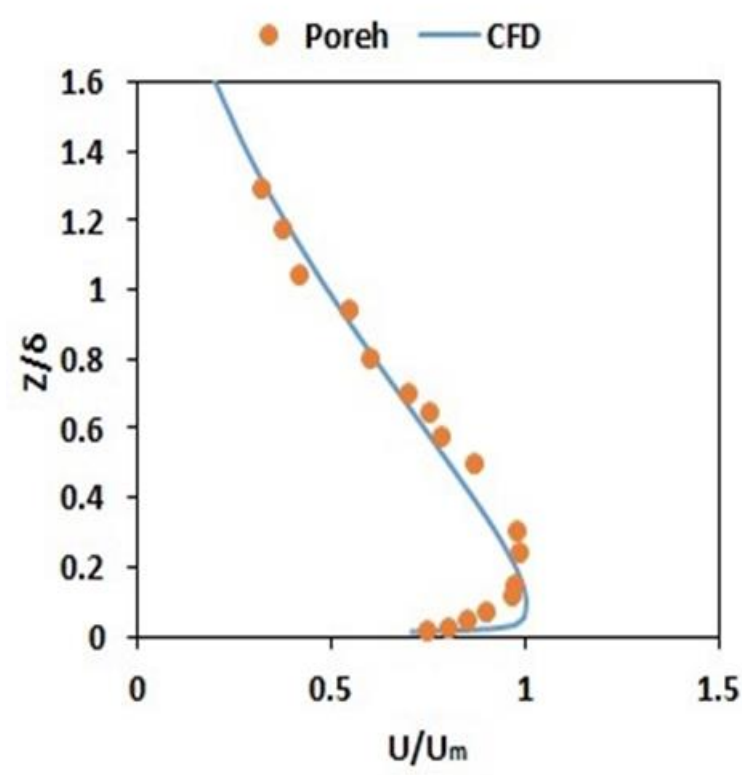

(c) $r / b=2$

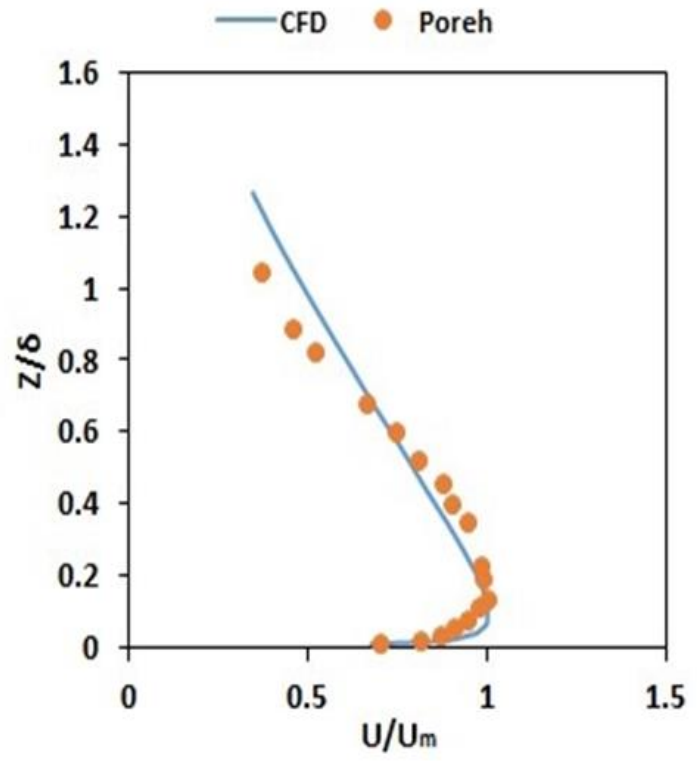

(b) $r / b=1.5$

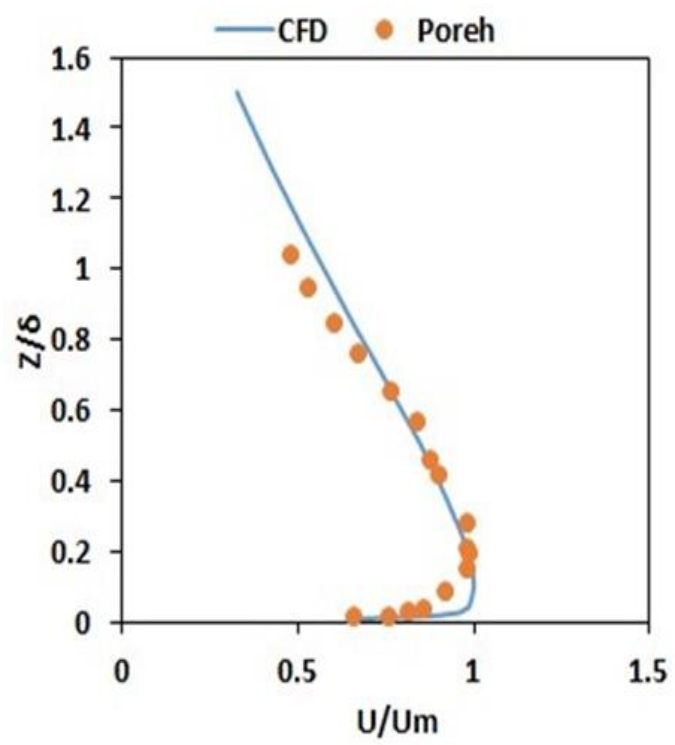

(d) $r / b=2.5$

Figure 9- Comparison of wall-normal distribution of radial wall jet velocity profiles at radial locations; (normalized scale) (a) $r / b=1 \quad$ (b) $r / b=1.5 \quad$ (c) $r / b=2$ (d) $r / b=2.5$

Figure 9 shows how the developed simulation was able to capture the radial wall jet at all four radial locations. The vertical distance $(\mathrm{z})$ is non-dimensionalized by the profile jet half-width and the velocity is non-dimensionalized by the profile maximum 
velocity. Discrepancies are observed near the wall, where problems are expected due to turbulence modeling limitations in the near wall region. Ultimately the quantities of interest are the maximum velocity $\left(U_{m}\right)$ and jet spread rate $(\delta)$ at the four radial locations, which are compared below:

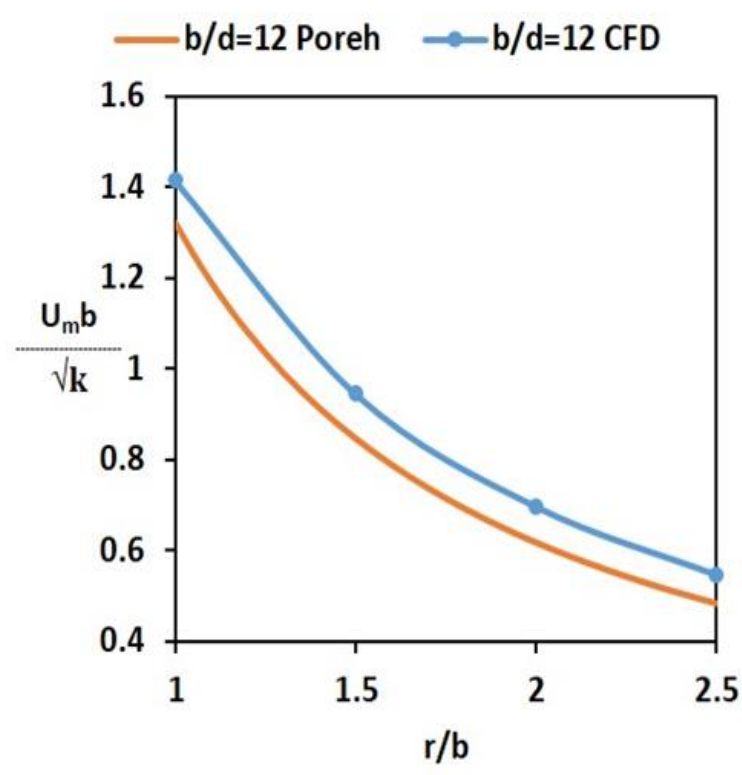

(a) $\frac{U_{\mathrm{m}} \mathrm{b}}{\sqrt{\mathrm{K}}} \operatorname{Vs} \mathbf{r} / \mathbf{b}$

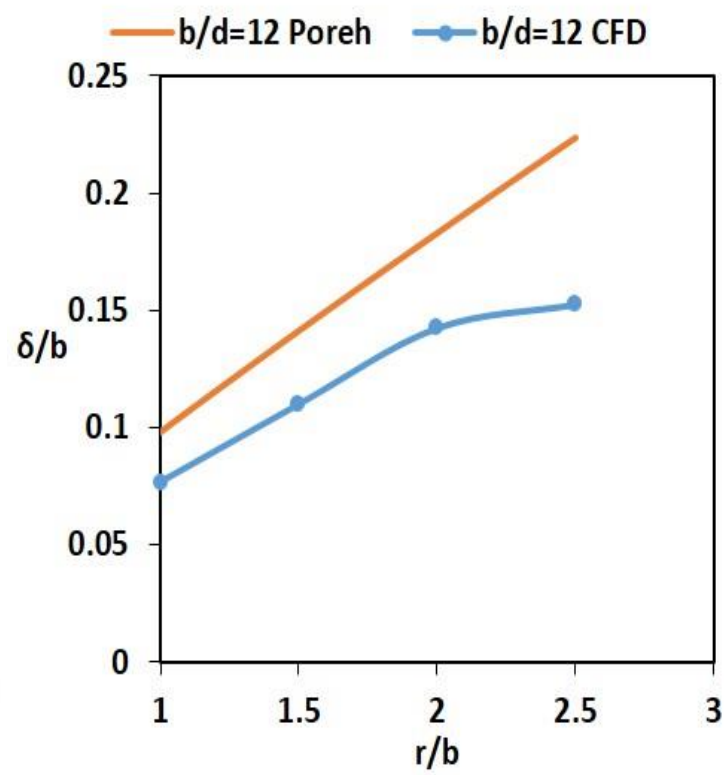

(b) $\delta /$ b Vs r/b

Figure $10-\frac{b}{D}=12$ Simulation Comparison of radial variation $(r / b)$ of $(a)$ non-dimensional radial wall jet maximum $\frac{U_{m} b}{\sqrt{K}}$ and $(b)$ non-dimensional radial wall jet thickness $\delta / b$ at four radial locations

In Figure 10 the maximum velocity is non-dimensionalized by the kinematic momentum and the jet half width is non-dimensionalized by nozzle to impingement distance. Figure 10 (a) shows reasonable agreement in the slope of decay of maximum jet velocities while the numerical values of current maximum radial jet velocity slightly over-predict Poreh's experimental data. There is about an $8 \%$ discrepancy in the simulation results which is considered reasonable within this engineering process. The spreading rate in Figure 10 (b) closely follows the Poreh's correlation and the overall trends are in reasonable agreement at all radial locations up to $r / b=2$. In addition, the 
current CFD data established a linear spreading rate over large range as that of Poreh's experimental data. Given that the simulation results are within an acceptable error of the experimental data, the simulation was re-run with the characteristic ratio of the PJMs:

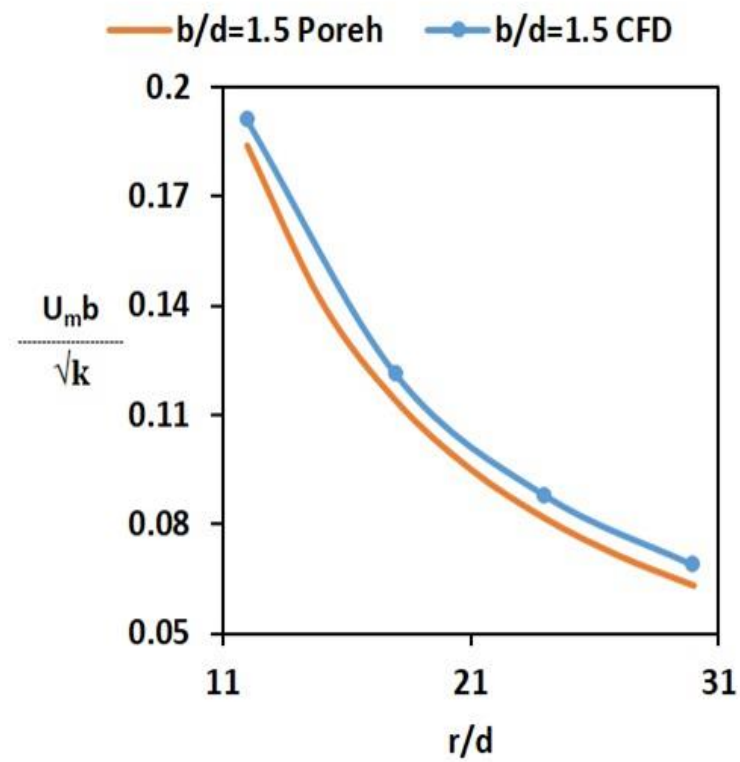

(a) $\frac{\mathbf{U}_{\mathbf{m}} \mathbf{b}}{\sqrt{\mathrm{K}}}$ Vs $\mathbf{r} / \mathbf{d}$

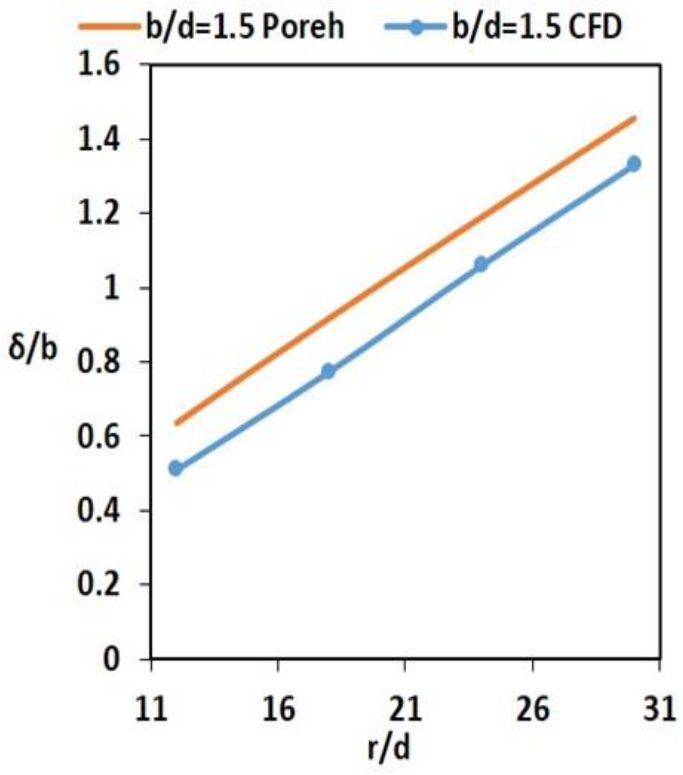

(b) $\delta /$ b Vs r/d

Figure $11-\frac{b}{D}=1.5$ Simulation Comparison of radial variation $(r / b)$ of $(a)$ non-dimensional radial wall jet maximum $\frac{U_{m} b}{\sqrt{K}}$ and (b) non-dimensional radial wall jet thickness $\delta / b$ at four radial locations

Figure 11 shows how Poreh's correlation evaluated at the PJM's characteristic ratio fairs against the CFD prediction. Both the simulation radial wall jet maximum velocity decay and the radial wall jet growth change at a similar rate as Poreh's correlation predicts. As far as magnitude is concerned, both $\delta$ and $U_{m}$ are in similar agreement with Poreh's correlation, as with the simulation conducted at $\frac{b}{D}=12$.Although this suggests that lowering the $\frac{b}{D}$ ratio doesn't affect the applicability of Poreh's correlations, the curved surface must be added in order to suggest their applicability under the PJM geometric condition. Because the impingement height $\mathrm{b}$ is much smaller, 
the non-dimensionalization of the $\mathrm{x}$ axis for this simulation is done with respect to the nozzle diameter. The data extraction locations are at the same physical location as those in Poreh's experiment.

\subsubsection{Curved Impingement Simulation of a Scaled Down PJM Model (b/D=1.5)}

Due to the geometric configuration of the PJM design, it is the case that a 2-D axis-symmetric simulation is no longer reasonable. The portion of domain that is axissymmetric is now a $3 \mathrm{D}$ volume and is outlined below:
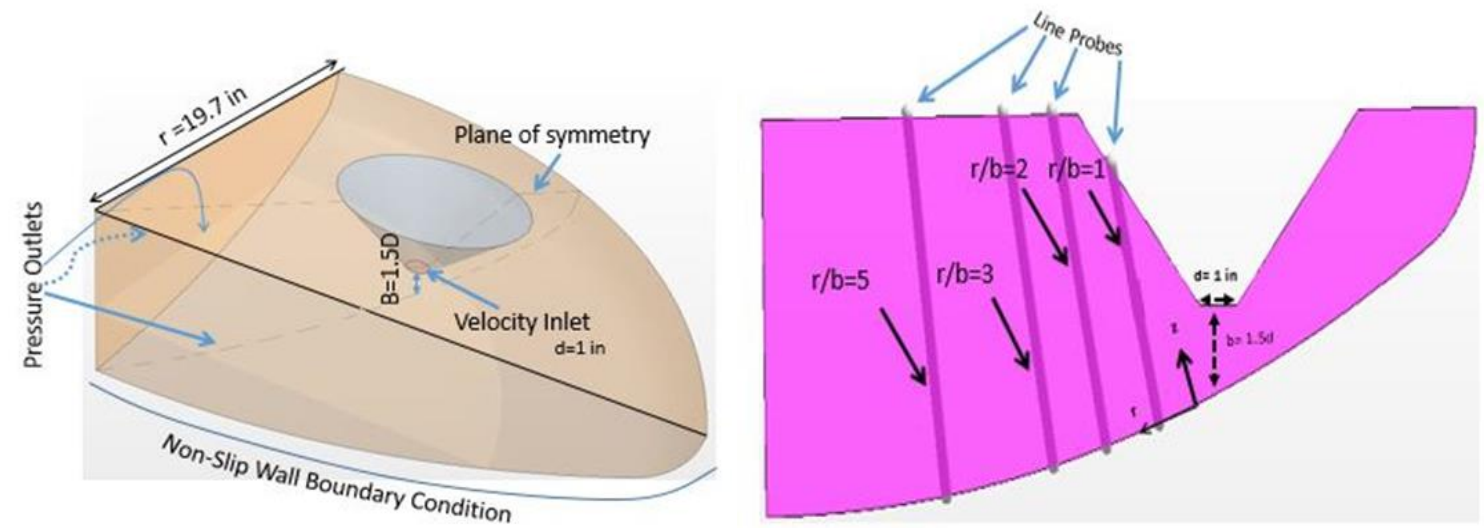

(a) 3D-quarter scaled PJM vessel geometry (b) 2D symmetric plane with probe lines

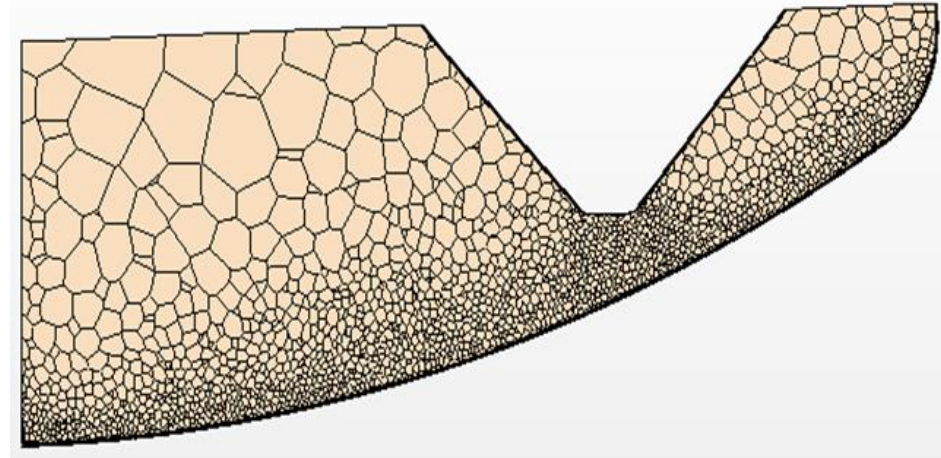

(c) Cross-section view of polyhedral mesh in the quarter scaled PJM vessel geometry

Figure 12- Schematic of PJM geometry and computational domain showing the geometrical dimensions with associated boundary conditions and the probe locations of radial velocity data extraction (a) $3 D$ quarter scaled down PJM vessel geometry (b) $2 D$ plane of symmetry on which data was sampled (c) Cross-section view of polyhedral mesh 
Figure 12 (a) shows the geometric configuration as well as the boundary conditions used in the final simulation. Clearly the impingement surface employs a curved geometry instead of conventional flat surface as Poreh's experiment. Figure 12 (c) shows the polyhedral mesh which is similar in nature to that of the 2D axis-symmetric simulations. Current experimental work on the PJM process uses water, so water is chosen as the fluid domain. The Reynolds number used in this simulation is similar to Poreh's experiment $\left(\approx 2 \times 10^{5}\right)$. The pressure outlets were kept at atmospheric pressure. The results from the 3D simulation are depicted below:

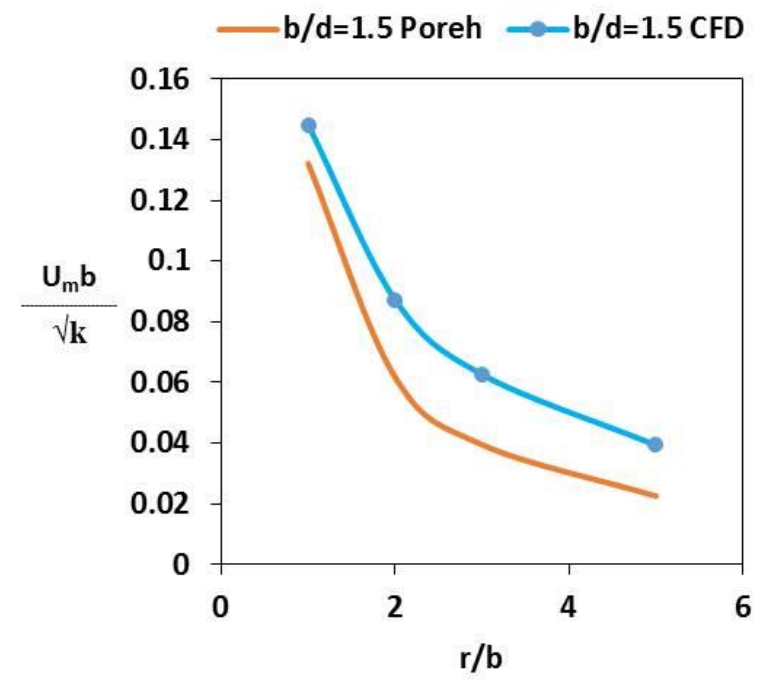

(a) $\frac{U_{m} b}{\sqrt{\bar{K}}}$ Vs r $/ d$

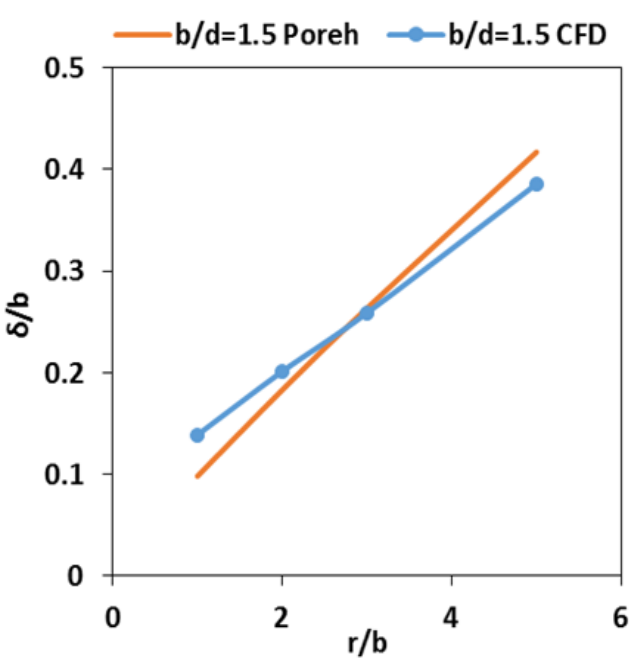

(b) $\delta / \mathrm{b} \mathrm{Vs} \mathrm{r/d}$

Figure 13- $\frac{b}{D}=1.5$ Curved impingement Simulation Comparison of radial variation $(r / b)$ of $(a)$ nondimensional radial wall jet maximum $\frac{U_{m} b}{\sqrt{K}}$ and (b) non-dimensional radial wall jet thickness $\delta / b$ at four radial locations

To examine the radial wall jet velocity profiles at the identical length scale range as of Poreh's study, the radial jet velocity (U) is investigated over the range of1 $\leq$ $\mathrm{r} / \mathrm{b} \leq 5$, which gives $1.5 \leq \mathrm{r} / \mathrm{d} \leq 7.5$, when normalized by jet diameter $(d)$. The radial variations $(r / b)$ of maximum radial wall jet velocity $\left(U_{m} b / \sqrt{K}\right)$ and radial wall jet 
spreading rate $(\delta)$ are displayed in Figure 13 (a) and (b) respectively. Figure 13 (a) shows steady decay of peak velocity with increasing radial distance. Interestingly, the decay of peak velocity is steeper initially up tor $/ b=2$, due to strong diffuser effects and then the rate of decay drastically reduces in the remaining large radial distance, since the flow passage again becomes nearly uniform. Overall agreement of radial distribution of maximum radial wall jet velocity with Poreh's correlation is quite satisfactory.

The spreading rate of the wall jet $(\delta)$ at various radial stations $(r / b)$ as plotted in Figure 13 (b) show a steadily increasing with increasing radial distances, confirming overall trend to fairly agree with Poreh's correlation giving similar linear spreading rate, although the growth rate are different, predicting higher value of lateral spreading at small radial distance, and, fall short at large radial locations. At small radial distance $(r / b \leq 3)$, the strong diffusing action delay the decay of radial velocity profiles at the free turbulent outer region resulting in thickening of the jets, leading to higher $\delta$, compared to jet flows in constant area passage described by Poreh, while, at large radial location $(r / b \geq 3)$, the decay of velocity at the outer region become faster due to lesser diffusing effects in relatively uniform flow passage. Based on the fact that the prediction of $\delta$ values are often scatter over a smaller range and confinement effect leads to slightly different value as also observed by Ghaneeizad et al [4], the current $\delta$ trends can be consider to be consistent with the Poreh's correlation.

Although the present PJM geometry has curved bottom surface which introduce confinement effects as well as diffuser action on the development of radial jet flows, the results demonstrate that the radial variation of the maximum local radial wall jet velocity $\left(U_{m}\right)$ and the spreading rate of wall jet $(\delta)$ can be well described by the Poreh's 
correlation and the later can be recommended for calculation jet parameters in practical PJM vessel design.

\subsection{Sparging of a Non-Newtonian Fluid}

The main focus of this sections work is to investigate the hydrodynamics of sparged non-Newtonian fluids. This will be realized by altering a validated CFD model. In order to obtain a reliable CFD model, a number of steps are taken to validate the final model through which sparging of a non-Newtonian fluid may be investigated. This is achieved by first validating a simulation of Sparged Newtonian fluid with existing experimental data. Then a second simulation will be conducted in which experimental data of power law fluids is matched. Once a degree of accuracy is reached within the validation process, rheological and physical characteristics of that simulation will be altered systematically and conclusions will be discerned.

\subsubsection{Replication of a Sparged Newtonian fluid}

Xe et al [52] conducted numerical simulations of bubble column flows in churnturbulent regimes using a Euler-Euler approach with an RNG $k-\varepsilon$ model. The numerical simulations matched time averaged radial gas volume fraction and axial velocity profiles with experimental data conducted by chen et al [54]. Xe's bubble column simulation was replicated using the geometry and boundary conditions depicted below: 


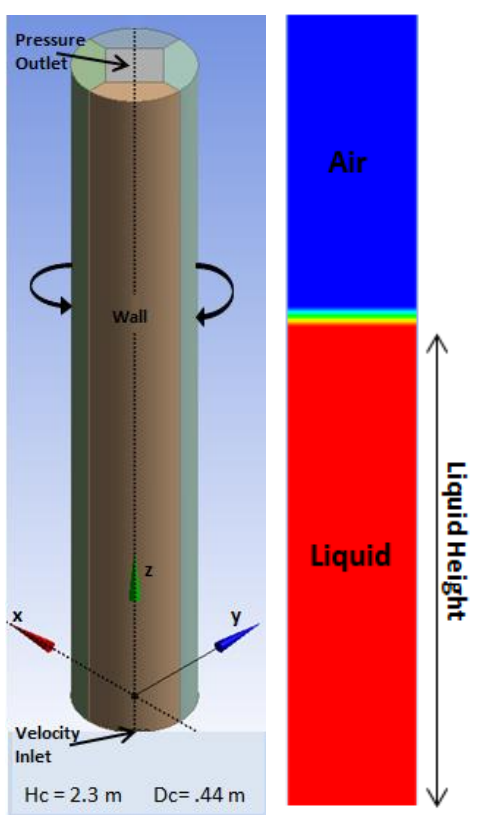

Figure 14- Boundary conditions, physical characteristic (Left,) and Initial conditions (Right) of Caixia Chen simulation

As can be seen in Figure 14, the $.44 \mathrm{~m}$ diameter column with a height of 2.3 meters has 3 boundary conditions, namely a velocity inlet, pressure outlet, and a non-slip wall. The liquid height in this experiment was kept at $1.1 \mathrm{~m}$. The simulation was run for 90 seconds with a time step of .01 seconds in order to reach a quasi-steady state. The results were obtained by time averaging field variables for the last 30 seconds, for a total simulation time of 120 seconds. Xe et al used the schiller neumann Drag model and thus was used in this replication. The same simulation was produced using different mesh sizes so that a mesh independent model could be declared. The comparison between the replicated simulation and chen's experimental data at one location in the bubble column is shown below: 

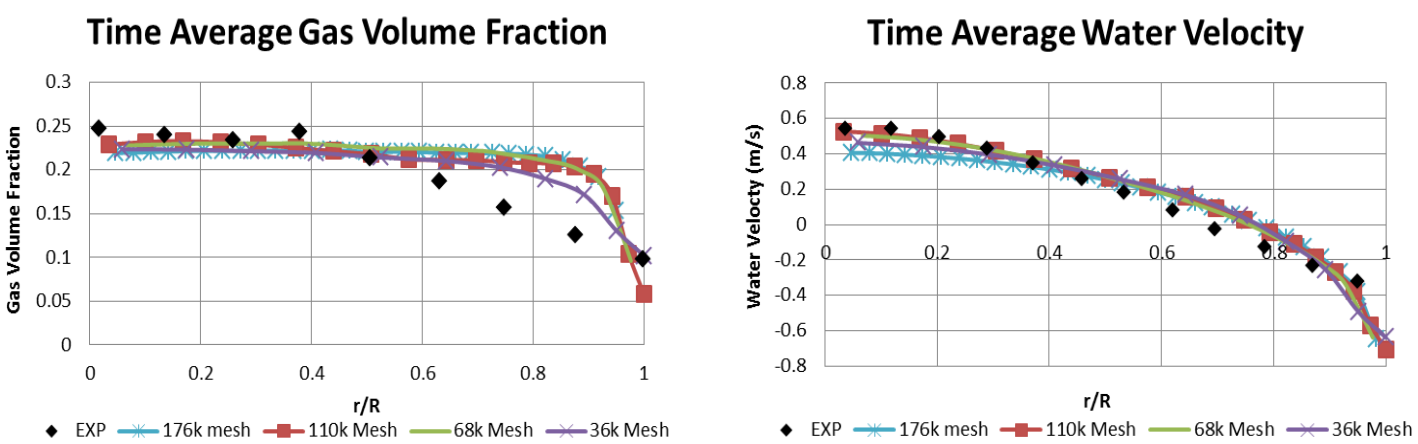

Figure 15-Replication of Xe's simulation results compared to chen's experimental data

From Figure 15 it can be observed that the model is mesh independent. The 36 thousand mesh count starts to deviate from the more refined meshes, so a 68 thousand mesh count is deemed as optimum. Furthermore, the comparison shows that the time averaged velocity is matched very well to the experimental, as is in Xe's simulation. The volume fraction profile of the simulation replication matches experimental data quantitatively well. Albeit, the profile shape of the volume fraction of the simulation has more of a plug profile. This discrepancy is also observed in Xe's work. The final mesh has 28 cells in the radial direction, 44 in the circumferential and 42 cells in the axial direction per meter, as is shown below:

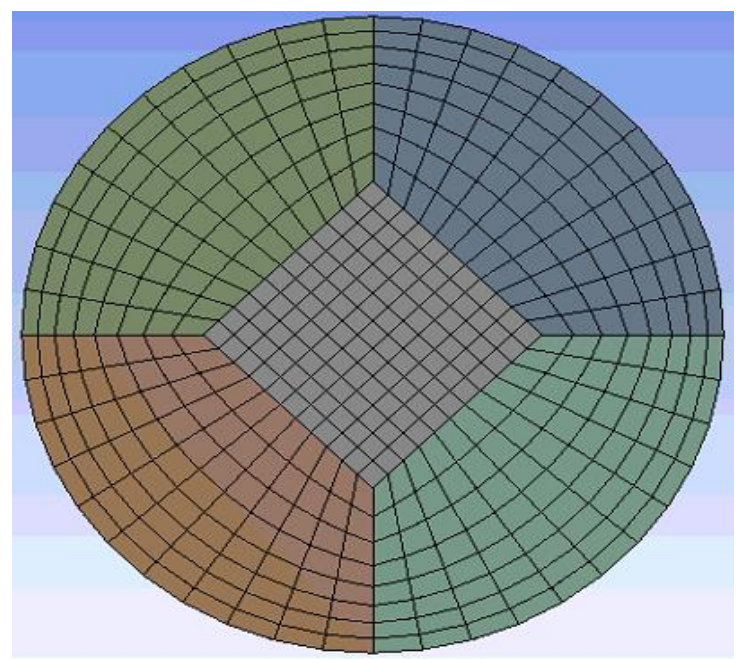

Figure 16- Cross section view of mesh for parametric study 
A uniform hexahedral conformal mesh is used, as other meshes tend to introduce instabilities as suggested by others who simulated Newtonian bubble columns [32, 37 , 55]. With a model that can capture the general physics of a bubble column operating with Newtonian fluid along with gained perspective of appropriate meshing and numerical methods, a model taking into account a non-Newtonian fluid may be initiated.

\subsubsection{Simulation of Bubble column operating with Non-Newtonian Fluid}

Amin et al [33] documented the effects of the power index on bubble columns with non-Newtonian fluids. Unlike the few other works on non-Newtonian bubble columns, Amin documented radial gas velocity and volume fraction profiles for four power law fluids with power indexes ranging from .98 to .21. For this reason Amin's work was chosen to be modeled. The characteristics of the fluid chosen to be modeled are listed below:

\begin{tabular}{ccccc}
\hline Name & Density $\left(\frac{\mathrm{kg}}{\mathrm{m}^{3}}\right)$ & $\mathbf{K}\left(\boldsymbol{P A} \boldsymbol{s}^{n}\right)$ & $\mathbf{n}$ & $\begin{array}{c}\text { Surface } \\
\text { Tension }\left(\frac{m N}{m}\right)\end{array}$ \\
\hline CMC & 995.65 & .32 & .68 & 73.92 \\
\hline
\end{tabular}

Table 4-Non-newtonian Fluid characteristics for validation simulation

The boundary conditions are the same as those listed in Figure 14 but with a diameter of $.292 \mathrm{~m}$ and a column height of $2.7 \mathrm{~m}$. The liquid height in the experiment is also $1.1 \mathrm{~m}$ while the inlet superficial gas velocities tested are $.0742 \frac{\mathrm{m}}{\mathrm{s}}$ and $.1981 \frac{\mathrm{m}}{\mathrm{s}}$. The simulation is also run with the same numerical discretization schemes and relaxation factors as the simulation of the Newtonian bubble column in the previous section. The only other alteration to the previous simulation is the rheological model. The Power Law for NonNewtonian Viscosity model in fluent was used. Below is a comparison between the experimental data Amin et al obtained and the simulation developed: 


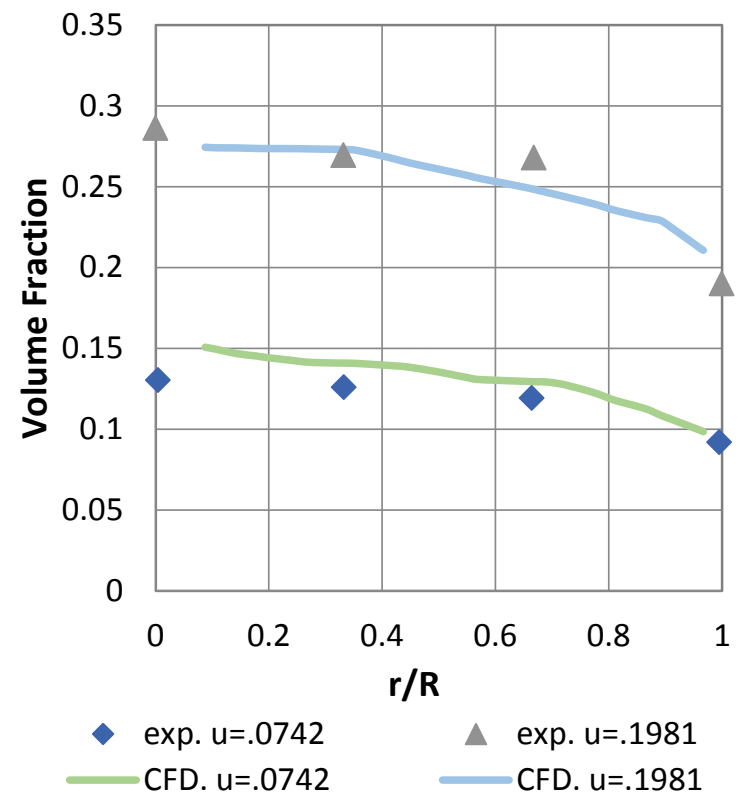

(a)
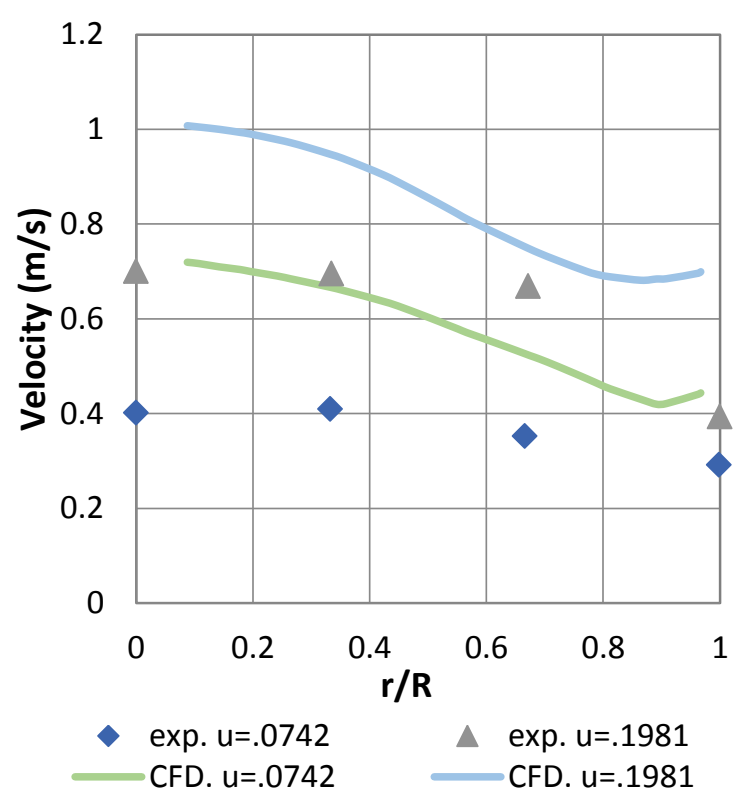

(b)

Figure 17- Circumferentially time averaged (a) air volume fraction and (b) air axial velocity profile simulation results compared to Amin's experimental data

Amin's experimental data for mean gas volume fraction and mean axial air velocity data extraction are both at a height of $45 \mathrm{~cm}$ above the inlet. It is observed in Figure 17 (a) that the volume fraction profiles predicted by the developed model are in good agreement with Amin's experimental data. The velocity profile on the other hand, is over predicted by $.3 \frac{\mathrm{m}}{\mathrm{s}}$ for both superficial gas velocities. Other drag and turbulence models were used in order to attempt to remedy the discrepancy but none proved fruitful. The purpose of this study is that of a qualitative investigation. Therefore, the analysis taken hereafter assumes the results to be representative of the flow despite this discrepancy.

Furthermore, grid refinement was conducted in the developed simulation in order to assure stability of the simulation: 


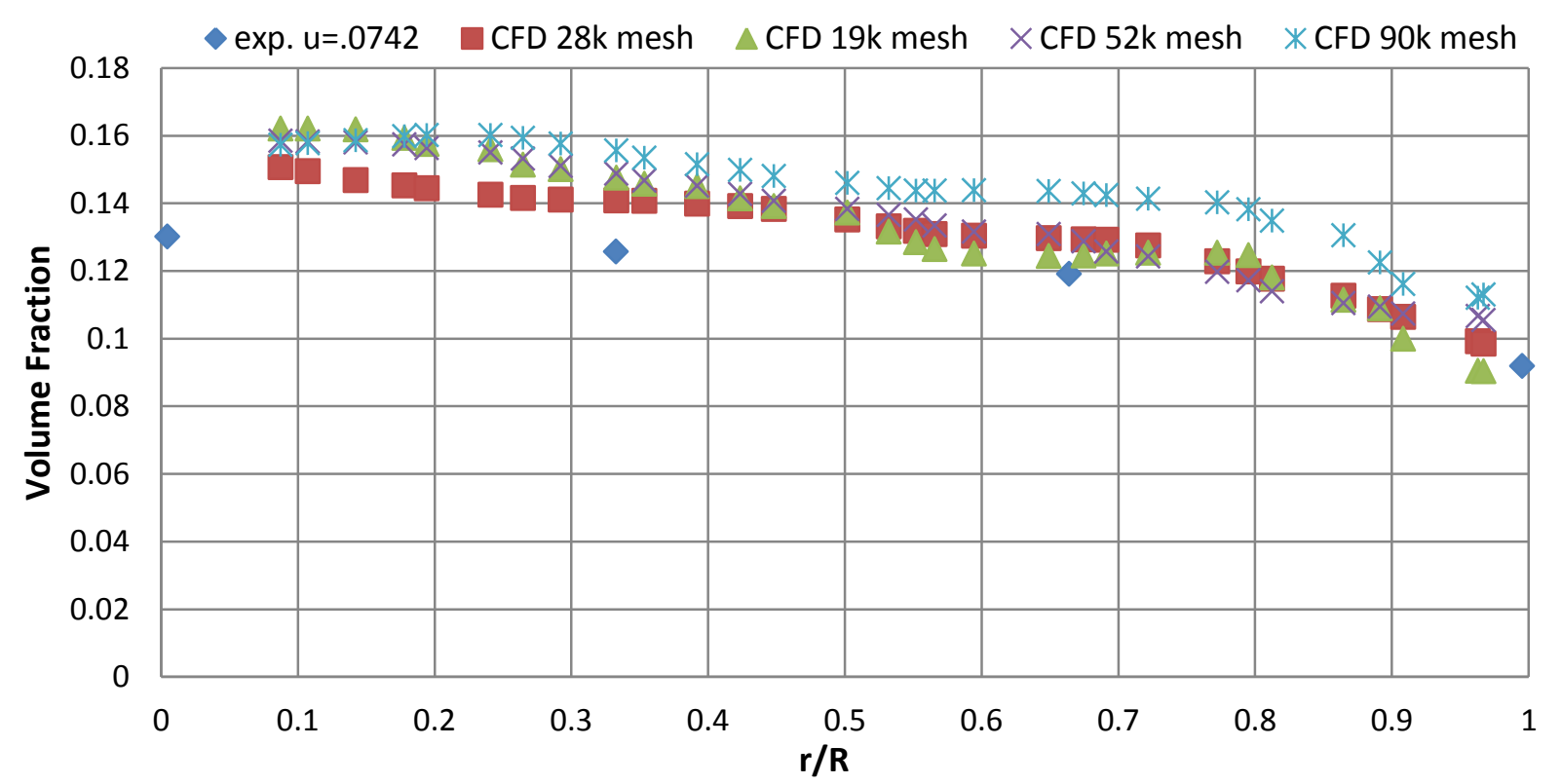

(a)

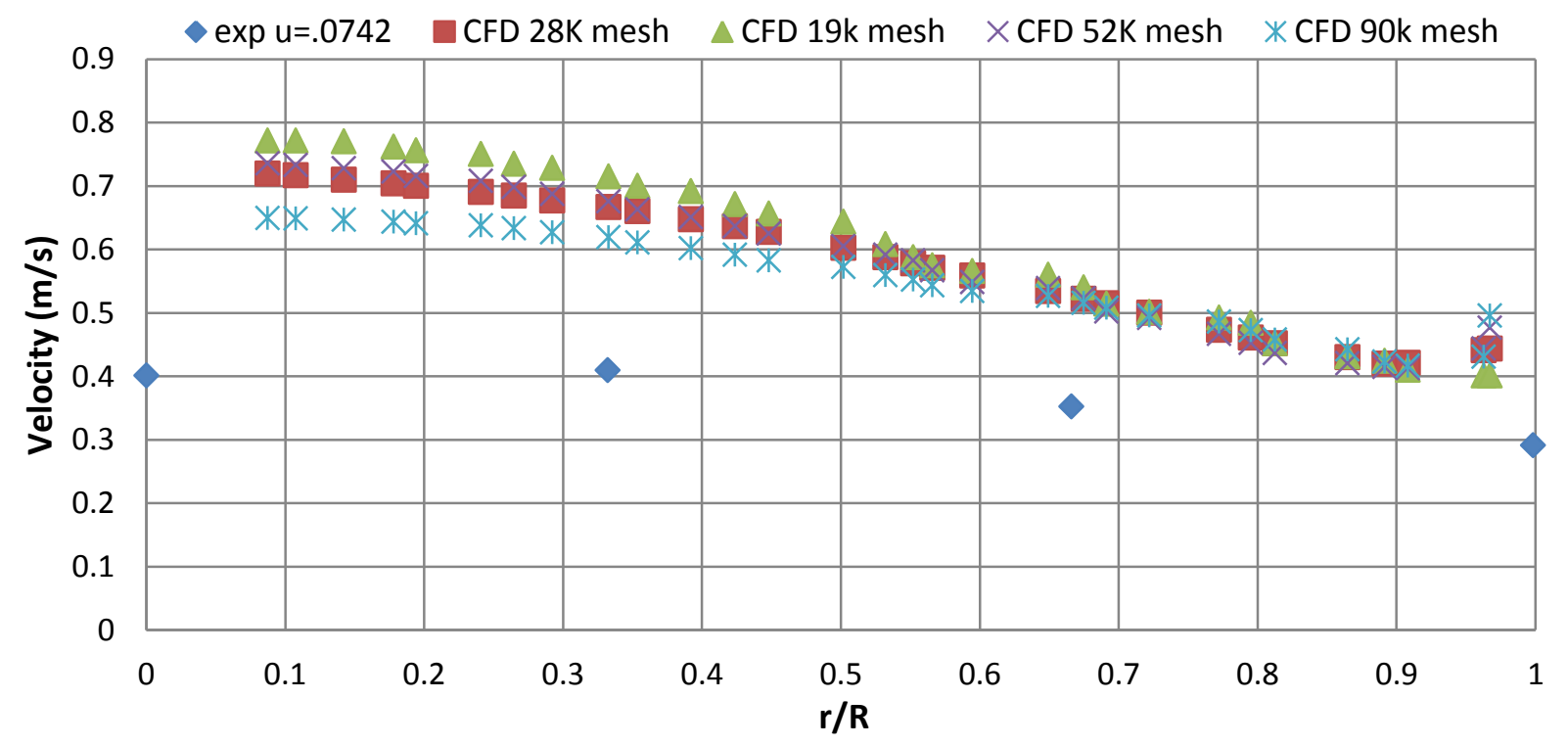

(b)

Figure 18- Developed simulation grid refinement study of the time averaged (a) air volume fraction (b) air axial velocity

Figure 18 shows that the variability in the simulation results is small with refining mesh, showing a degree of robustness in the simulation. The simulation also is tested for time averaged convergence: 


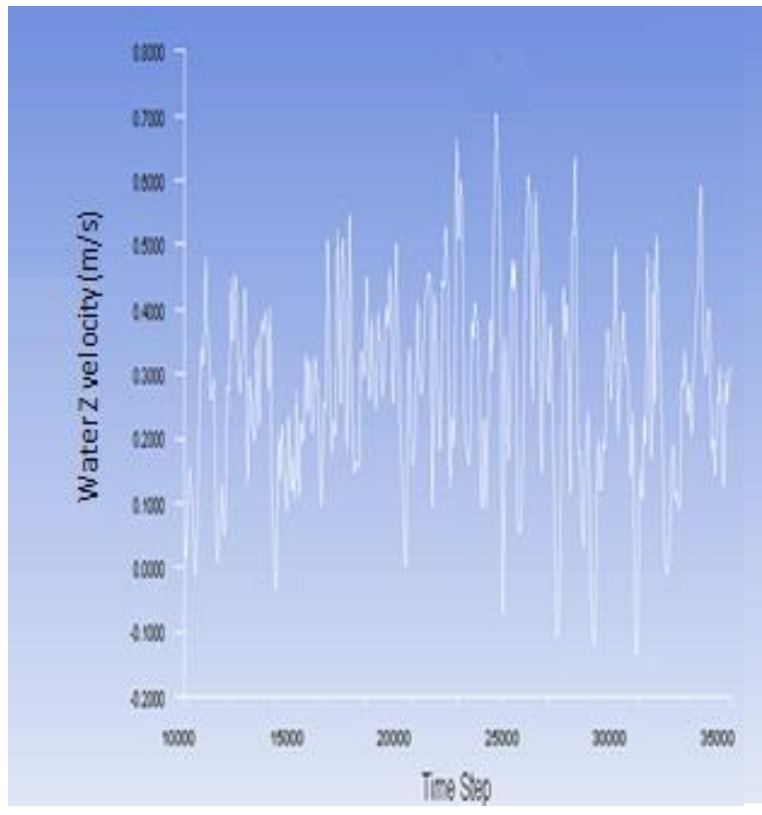

(a)

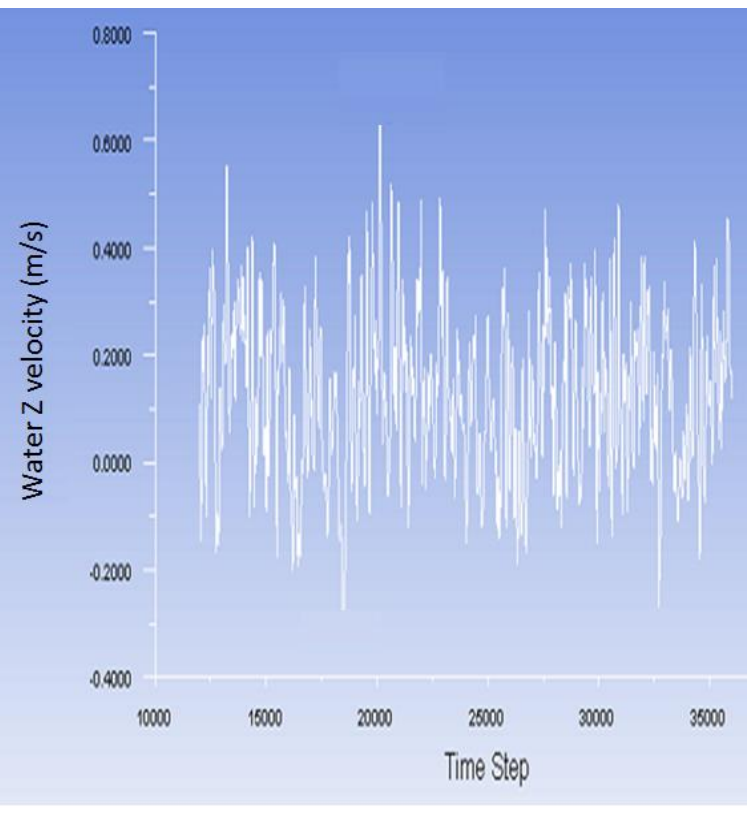

(b)

Figure 19- Instantaneous velocity at the center of the bubble column at a height of (a) $40 \%$ of liquid height and (b) $80 \%$ of liquid height

In figure 19 the water velocity at a point is observed to fluctuate at a height of (a) $40 \%$ of the liquid height and (b) $80 \%$ of the liquid height. The instantaneous water velocity is plotted from 120 seconds to 240 seconds. We see that although the instantaneous velocity changes with time there is a general trend of oscillation. The magnitude of oscillation also appears to be transient, but most of the oscillations are near the $.5 \mathrm{~m} / \mathrm{s}$ and $.4 \mathrm{~m} / \mathrm{s}$ mark.

Given that the simulation results 1) qualitatively match Amin's experimental data and are quantitatively within reasonable error, 2) are fairly stable to grid refinements, and 3) are near a quasi-steady state, it is concluded that the simulation can substantiate meaningful qualitative results during a parametric study. 


\subsubsection{Parametric study Simulation of Bubble column operating with Non-Newtonian Fluid}

As mentioned previously, there are no studies concerning the effects that $\frac{H}{D}$ have on the volume fraction and velocity profile of non-Newtonian Sparged columns.

Particularly, no information on $\frac{H}{D}$ values as low as those observed in the waste tanks are studied. Furthermore, the radial gas volume fraction and axial velocity sensibility to changes with index coefficients at different $\frac{H}{D}$ are also non-existing, to the authors knowledge. Lastly, none of the available literature studies the event that the power index is greater than one. In light of these facts the validated simulation will be ran with varying characteristic ratio numbers $\left(\frac{H}{D}=5.2-.34\right)$ and study the resulting effects. Following this procedure the power law index will be varied and evaluated at two different characteristic ratio numbers.

Non-Newtonian $\frac{H}{D}$ Study

The simulation developed was run with an inlet air velocity of .1942 $\frac{\mathrm{m}}{\mathrm{S}}$ along with the same diameter of Amin's et al experiment. In order to alter the $\frac{H}{D}$ ratio the height of the column was changed while the diameter held constant. The distance between the height of the liquid and the outlet pressure boundary condition was kept at a constant of $1.2 \mathrm{~m}$. Like the previous simulations, time averaging was conducted for 30 seconds after waiting 90 seconds for quasi-steady state to be reached. The extraction of data was gathered at $40 \%$ and $80 \%$ of the liquid height, scaling with the different $\frac{H}{D}$ ratios. The following results ensued: 


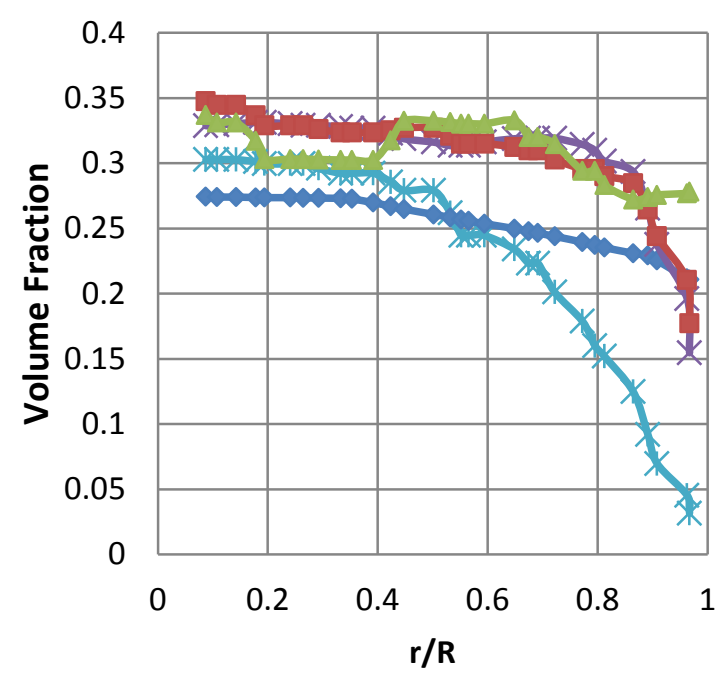

$\because \mathrm{H} / \mathrm{D}=5.2 \multimap \mathrm{H} / \mathrm{D}=3.8 \longrightarrow \mathrm{H} / \mathrm{D}=2.4$

$-\mathrm{H} / \mathrm{D}=1.37 \rightarrow \mathrm{H} / \mathrm{D}=.34$

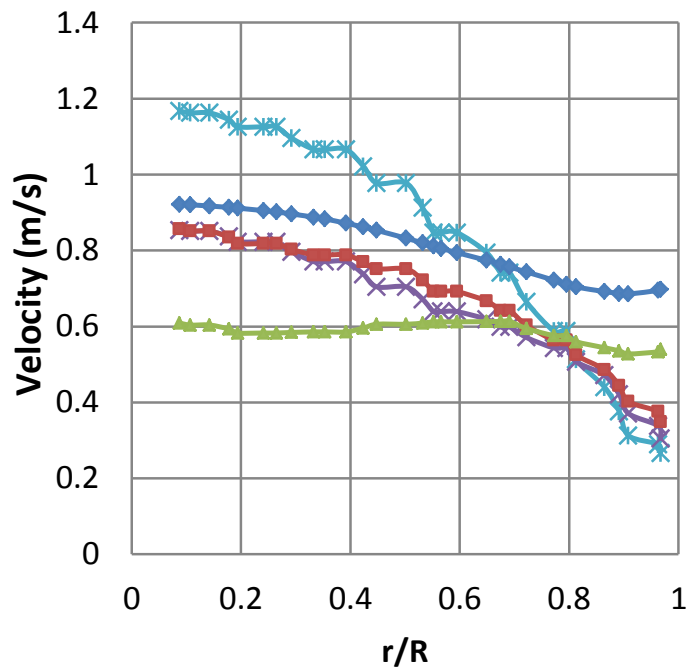

$\because \mathrm{H} / \mathrm{D}=5.2 \longrightarrow \mathrm{H} / \mathrm{D}=3.8 \longrightarrow \mathrm{H} / \mathrm{D}=2.4$

$\longrightarrow H / D=1.37 \multimap H / D=.34$

(a)

(b)

Figure 20- H/D comparison ( $\frac{H}{D}=5.2-.34$ ) of (a) air volume fraction and (b) air axial velocity at $40 \%$ of the liquid height

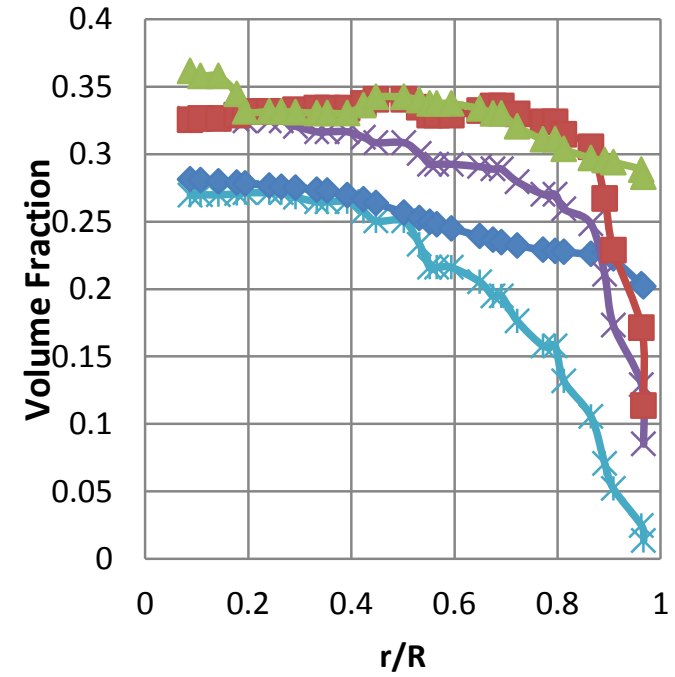

$\because \mathrm{H} / \mathrm{D}=5.2 \multimap \mathrm{H} / \mathrm{D}=3.8 \sim \mathrm{H} / \mathrm{D}=2.4$

$-H / D=1.37-H / D=.34$

(a)

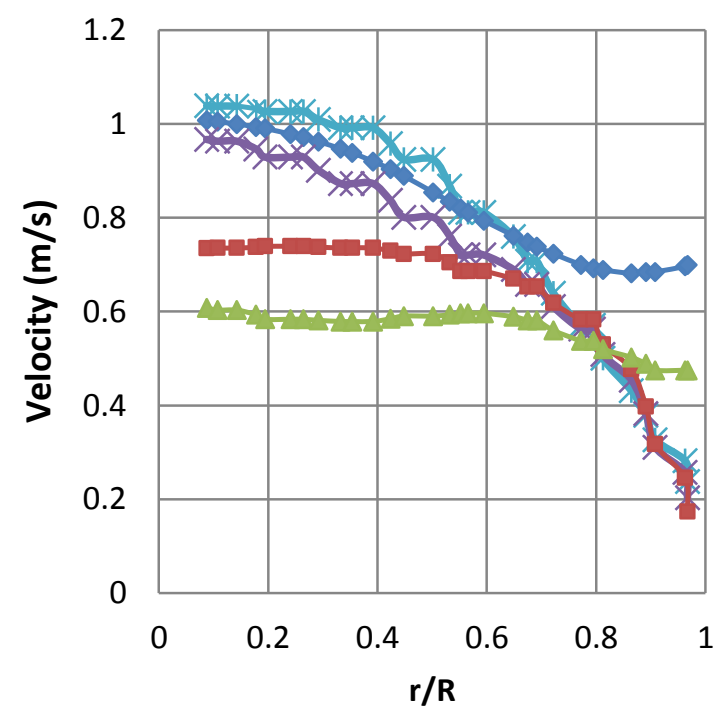

米 $\mathrm{H} / \mathrm{D}=5.2 \longrightarrow \mathrm{H} / \mathrm{D}=3.8 \longrightarrow \mathrm{H} / \mathrm{D}=2.4$

$\longrightarrow \mathrm{H} / \mathrm{D}=1.37 \rightarrow \mathrm{H} / \mathrm{D}=.34$

(b)

Figure 21- H/D comparison ( $\left.\frac{H}{D}=5.2-.34\right)$ of (a) air volume fraction and (b) air axial velocity at $80 \%$ of the liquid height 
From Figure 21 (a) it is observed that the volume fraction profile becomes flatter with decreasing $\frac{\mathrm{H}}{\mathrm{D}}$ ratio. At $\frac{H}{D}=5.2$ the most parabolic profile is observed while at $\frac{H}{D}=.34$ the volume fraction is almost flat. A similar trend can be observed in Figure 21 (a). This is due to the fact that as the height of the fluid is reduced the extraction of data occurs much closer to the inlet. A look at Figure 22 showing trends of the air volume fraction profile at a $2 \mathrm{D}$ cross-section provides evidence for this hypothesis.

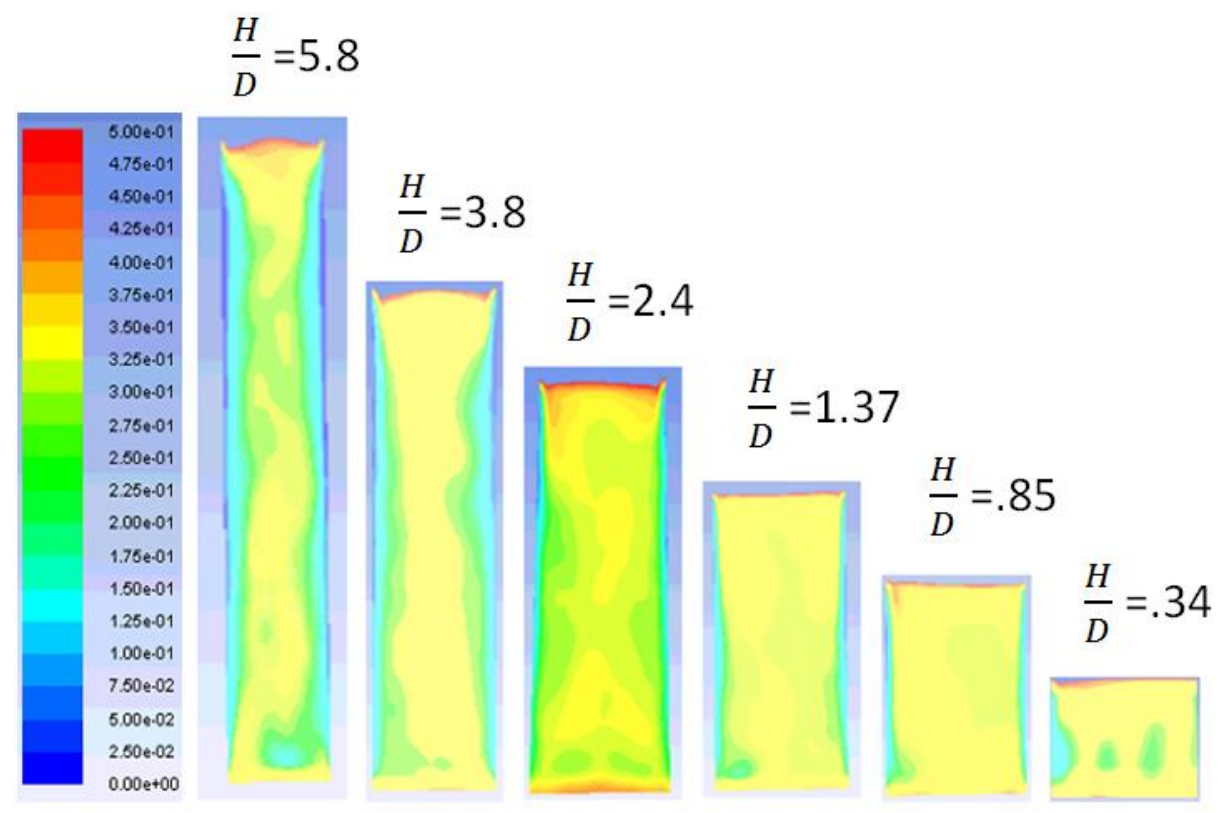

Figure 22- Time averaged air volume fraction profile across a $2 D$ plane for $H / D=5.8-.34$ with a clip to range of .5 (not to scale)

It is observed that in all simulations the air volume fraction near the inlet has a plug profile. Immediately after this plug profile the volume fraction tends to concentrate itself near the walls. The volume fraction then tends to a developed state where the concentration of air is higher at the center. It is important to note that Figure 22 is time averaged for 30 seconds and that an instantaneous gas volume profile would look entirely different, as the volume fraction plume oscillates from side to side as shown in previous studies. From this one concludes that the volume fraction profile expected in both 
magnitude and shape are in part a function of where they are extracted in reference to the inlet. This reference distance to the inlet is a percentage of the column diameter, giving significance to the $\frac{H}{D}$ parameter.

When studying the behavior of the air velocity profile in Figure 21 (a) and Figure 21 (b), one can see similar flattening of the axial air velocity profile with decreasing $\frac{H}{D}$. The first noticeable outcome is that the center velocity tends to increase when comparing the same $\frac{H}{D}$ at $40 \%$ liquid height to $80 \%$ liquid height. This is due to that fact that the bubbles haven't yet reach terminal upward velocity, so it is expected to see some velocity increase. For this same reason the velocity tends to be higher with increasing $\frac{H}{D}$, since the height of the fluid column is the parameter increasing, giving more time for acceleration to take place. The behavior near the wall of the air velocity profiles has a noticeable trend. As the velocity profile is given more time to become developed the effect of the wall is more prominent, giving lower velocities due to the no slip boundary condition at the wall. The noticeable outlier in this trend is the curve formed by $\frac{H}{D}=2.4$.

Lastly, the time averaged volume fraction profiles on a 2D contour surface whose normal is perpendicular to the axial direction was investigated. Below are the time averaged contour plots of air volume fraction: 


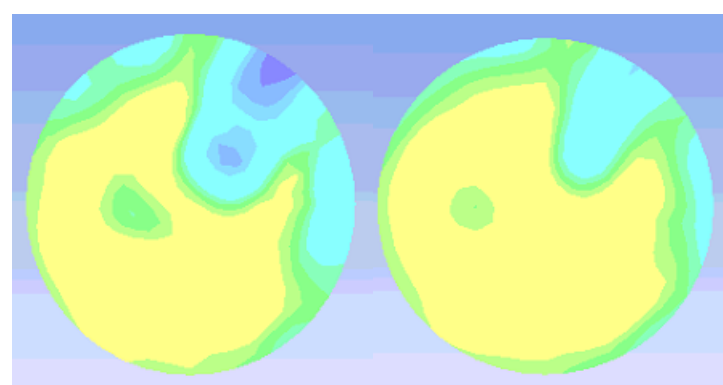

(a1) (a2)

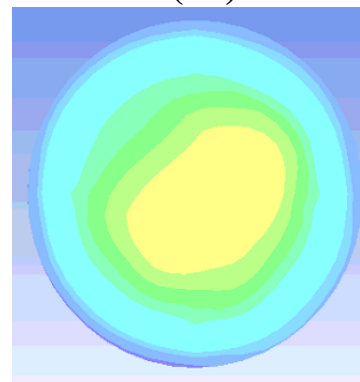

(c1)

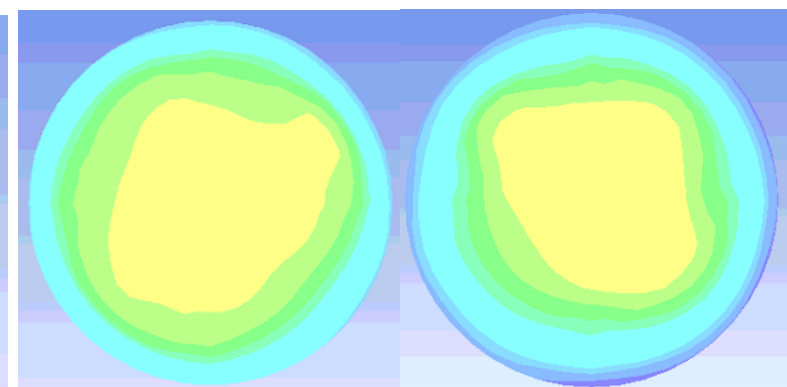

(b1)

(b2)

Figure 23- Time averaged air volume fraction on an axial section view for $(a) \frac{H}{D}=.34,(b) \frac{H}{D}=3.8,(c)$ $\frac{H}{D}=5.8$ at (1) $40 \%$ and (2) $80 \%$ of the liquid height

It is observed that there is little difference, from a qualitative point of view, between the time averaged air volume fraction contour plots at $40 \%$ and $80 \%$ of liquid height. There is a tendency for the volume fraction profile to concentrate near the center and lessen in concentration radially outward until the column wall boundary condition is reached. This behavior is observed in experimental data. In both Figure 23 al and a2, this tendency is not observed. This could be due to the fact that at an $\frac{H}{D}$ ratio of .35 the data is extracted too close to the inlet velocity, not giving enough time for wall effects take precedence.

\section{Non-Newtonian $n$ Study}

The validated simulation was run with four different power index coefficients less than one and at two $\frac{H}{D}$ ratios. Below are the resulting profiles: 


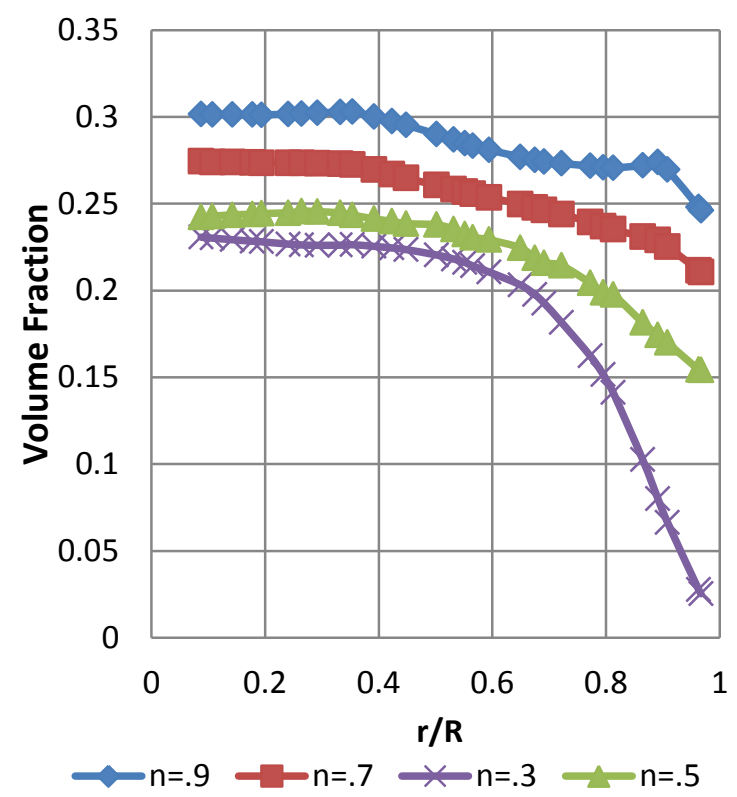

(a)

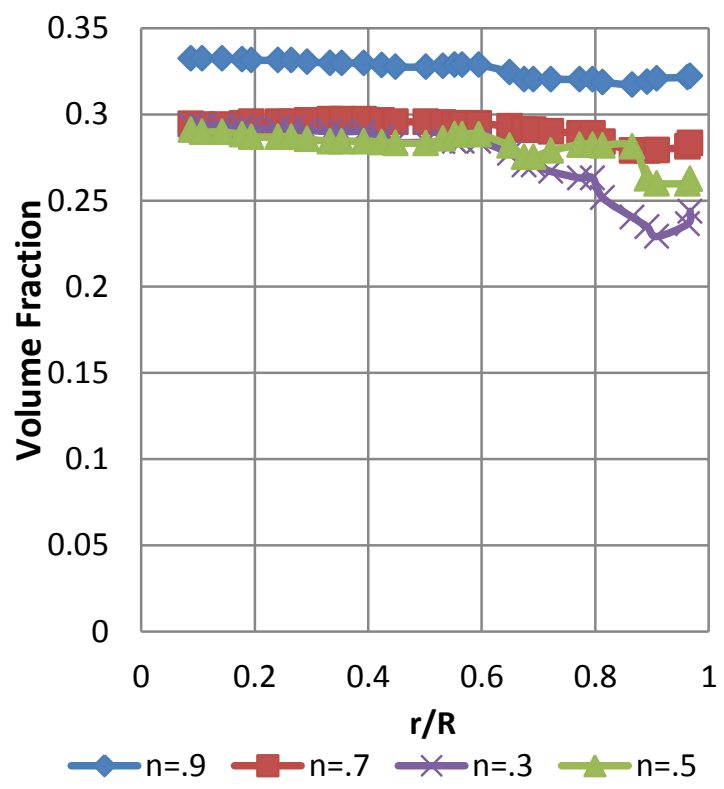

(b)

Figure 24- Power coefficient study on volume fraction for $n$ less than one $(n=.9, .7, .5, .3)$ at $\frac{H}{D}$ ratios of (a) 3.8 and (b) .34

It can be observed in Figure 24 (a) and (b) that with decreasing index coefficient

that the volume fraction profiles become more parabolic. The effect of decreasing $\frac{H}{D}$ ratio is clearly seen in the flattening of the profiles, yet volume fraction near the walls appears to decrease. This is likely due to the decrease in viscosity near the center where velocity is higher.

Lastly the effects of increasing the power coefficient above one on the volume fraction and velocity profile were investigated. This was similarly accomplished by running the validated simulation at three different power index ratios. The results are shown below: 


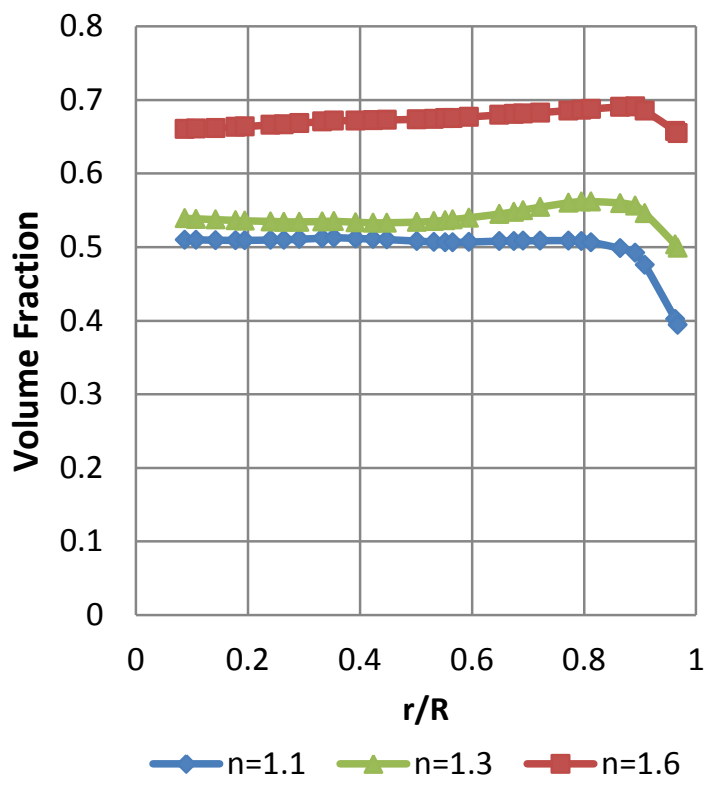

(a)

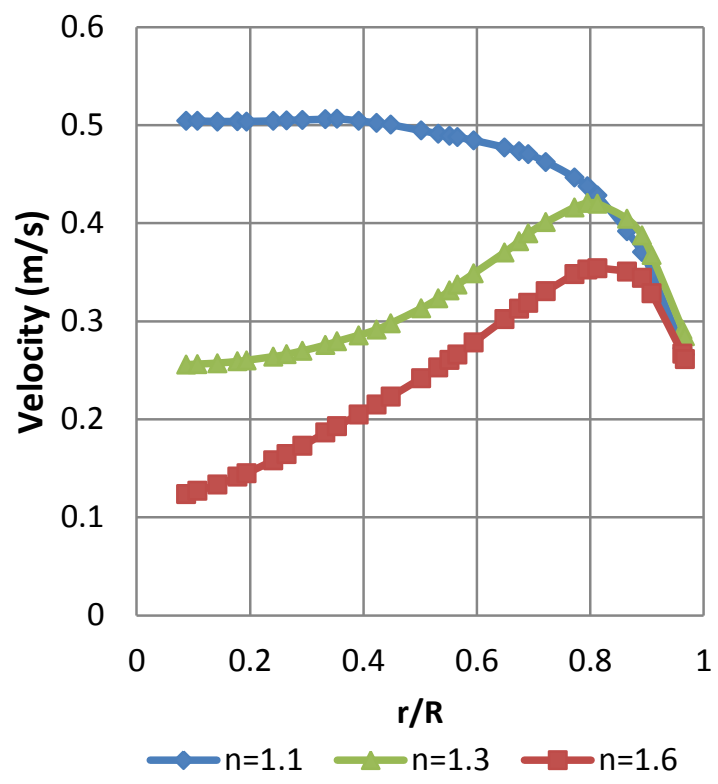

(b)

Figure 25- Power coefficient study on (a) volume fraction and (b) velocity for $n$ greater than one $(n=1.1$, $1.3,1.6$,) at an $\frac{H}{D}$ ratio of 3.8

The effects of increasing the power index coefficient to a number greater than one on the volume fraction results in increased volume fraction as can be observed in Figure 25 (a). The resulting velocity profile on Figure 25 (b) indicates a decrease in velocity with increasing power coefficient. A possibility could be that an increase in viscosity limits the terminal velocity of the bubbles near the center of the column, resulting in higher concentrations of bubbles. 


\section{CHAPTER V - CONCLUSIONS AND FUTURE CONSIDERATIONS}

The Pulse Jet Mixing process is a vital part of the nuclear waste deactivation and decommissioning mission at DOE's Hanford site. Areas of uncertainty within the PJM process are numerous. This thesis investigates two areas of uncertainty within this process, and they are 1) the applicability of using Poreh's correlations to describe the radial wall jets created in the PJM process and 2) aspects of the hydrodynamics involved with using sparging within the waste tanks.

\subsection{Applicability of Poreh's Correlation}

Poreh's correlations describing the radial wall jet after impingement were concluded to be largely appropriate for their use in the PJM process. This was accomplished through a validation model where the general physics and Poreh's experimental data was captured and then used to assess the effects of lowering the characteristic ratio $(b / d)$ to that of the PJMs. The simulation suggested that the low characteristic ratio did not affect the correlations accuracy. Applicability was then further investigated by looking into the effects of adding a curved impingement surface. This involved the use of a 3D model which showed to have the largest impact on the results. Comparing the final simulation results to Poreh's correlation suggested the correlation to be a good approximation of the radial wall jet under low characteristic ratio and curved impingement surface. This suggestion aids in the confidence and validation of the use of such correlations in the current analytical work for the PJM process.

Substantial future work in this area can be pursued. The nuclear waste at Hanford has been classified as a non-Newtonian fluid. An investigation on the effects that adding non-Newtonian fluid characteristics have on the velocity profile of the radial wall jet 
would be interesting. Furthermore, the radial wall jet profile characteristics are important in determining the rate and extent of erosion in the PJM process. This is an area of concern due to the presence of a large particle size distribution in the waste tanks.

Repeated use of the PJM process in the waste tanks could quickly erode the impingement surface of the waste tanks. Finally, an investigation on the effect of having multiple jets on Poreh's correlation would also be recommended for proper validation.

\subsection{Sparging of a Non-Newtonian Fluid}

With regards to sparging of the waste tanks, the effect of the characteristic ratio (

$\frac{\mathrm{H}}{\mathrm{D}}$ ) and power law index on bubble columns with non-Newtonian fluids was investigated. It was observed that a flattening of the profile occurs with decreasing $\frac{\mathrm{H}}{\mathrm{D}}$ ratio for both air volume fraction and air axial velocity. This is predicted to be as a result of how close the outlet is to the inlet. The wider the diameter of the column through which sparging occurs the longer vertical distance required for the flow to become fully developed. It is therefore expected that if the PJMs were sparged, they would experience a plug air volume fraction and axial velocity profile. Furthermore, a decrease in the power law index coefficient was associated with a more parabolic profile independent of the $\frac{\mathrm{H}}{\mathrm{D}}$ ratio. Lastly, an increase in power law index coefficient was associated with an increase in volume fraction.

Sparging in the area of non-Newtonian fluids has not been thoroughly investigated. Future work in this area that would be beneficial to the design and development of the PJM process is to investigate the effects that altering rheological and physical characteristics of the flow would have on the mixing state of the column. This 
would more directly address the ultimate intent of the PJM process. Furthermore, the synergistic effects on mixing that both sparging and jet impingement has on mixing would be an interesting topic. 


\section{$\underline{\text { REFERENCES }}$}

1. Chris Guenther, M.S., William Rogers, Sofiane Benyahia, Rahul Garg, Tingwen Li, TECHNICAL REPORT ON NETL'S NON-NEWTONIAN MULTIPHASE SLURRY WORKSHOP 2013, NETL: Morgan Town, WV . p. 1-65.

2. Poreh, M., Y.G. Tsuei, and J.E. Cermak, Investigation of a Turbulent Radial Wall Jet. Journal of Applied Mechanics, 1967. 34(2): p. 457-463.

3. Zuckerman, N. and N. Lior, Impingement Heat Transfer: Correlations and Numerical Modeling. Journal of Heat Transfer, 2005. 127(5): p. 544-552.

4. Ghaneeizad, S.M., J.F. Atkinson, and S.J. Bennett, Effect of flow confinement on the hydrodynamics of circular impinging jets: implications for erosion assessment. Environmental Fluid Mechanics, 2015. 15(1): p. 1-25.

5. Sengupta, A. and P.P. Sarkar, Experimental measurement and numerical simulation of an impinging jet with application to thunderstorm microburst winds. Journal of Wind Engineering and Industrial Aerodynamics, 2008. 96(3): p. 345365 .

6. B. Rosendall, C.B., F. Wen and K. Knight Validating CFD models of multiphase mixing in the waste treatment plant at the Hanford site, in $14^{\text {th }}$ International Conference on Nuclear Engineering 2006: Miami Floriada, USA.

7. Beltaos, S. and N. Rajaratnam, Impinging circular turbulent jets. Journal of the hydraulics division, 1974. 100(10): p. 1313-1328.

8. Cooper, D., et al., Impinging jet studies for turbulence model assessment-I. Flow-field experiments. International Journal of Heat and Mass Transfer, 1993. 36(10): p. 2675-2684.

9. Craft, T.J., L.J.W. Graham, and B.E. Launder, Impinging jet studies for turbulence model assessment-II. An examination of the performance of four turbulence models. International Journal of Heat and Mass Transfer, 1993. 36(10): p. 2685-2697.

10. Jambunathan, K., et al., A review of heat transfer data for single circular jet impingement. International Journal of Heat and Fluid Flow, 1992. 13(2): p. 106115 . 
11. Baydar, E. and Y. Ozmen, An experimental investigation on flow structures of confined and unconfined impinging air jets. Heat and Mass Transfer, 2006. 42(4): p. 338-346.

12. Fairweather, M. and G. Hargrave, Experimental investigation of an axisymmetric, impinging turbulent jet. 2. scalar field. Experiments in fluids, 2002. 33(4): p. 539544.

13. Glauert, M., The wall jet. Journal of Fluid Mechanics, 1956. 1(06): p. 625-643.

14. Beltaos, S. and N. Rajaratnam, IMPINGEMENT OF AXISYMMETRIC DEVELOPING JETS. Journal of Hydraulic Research, 1977. 15(4): p. 311-326.

15. Wood, G.S., et al., Physical and numerical modelling of thunderstorm downbursts. Journal of Wind Engineering and Industrial Aerodynamics, 2001. 89(6): p. 535-552.

16. Fairweather, M. and G. Hargrave, Experimental investigation of an axisymmetric, impinging turbulent jet. 1. Velocity field. Experiments in Fluids, 2002. 33(3): p. 464-471.

17. Picardi, R.N., Numerical Analysis of Multiphase Flow in Bubble Columns and Applications for Microbial Fuel Cells, in Mechanical Engineering. 2015, Virginia Tech: 2015-04-15.

18. Attfield, P.V., Stress tolerance: the key to effective strains of industrial baker's yeast. Nature biotechnology, 1997. 15(13): p. 1351-1357.

19. Berovic, M. and M. Legisa, Citric acid production. Biotechnology Annual Review, 2007. 13: p. 303-343.

20. Hermann, T., Industrial production of amino acids by coryneform bacteria. Journal of biotechnology, 2003. 104(1): p. 155-172.

21. Strik, D.P., et al., Renewable sustainable biocatalyzed electricity production in a photosynthetic algal microbial fuel cell (PAMFC). Applied microbiology and biotechnology, 2008. 81(4): p. 659-668.

22. Kantarci, N., F. Borak, and K.O. Ulgen, Bubble column reactors. Process Biochemistry, 2005. 40(7): p. 2263-2283. 
23. Hekmat, A., A.E. Amooghin, and M.K. Moraveji, CFD simulation of gas-liquid flow behaviour in an air-lift reactor: determination of the optimum distance of the draft tube. Simulation Modelling Practice and Theory, 2010. 18(7): p. 927-945.

24. Rampure, M.R., V.V. Buwa, and V.V. Ranade, Modelling of Gas-Liquid/GasLiquid-Solid Flows in Bubble Columns: Experiments and CFD Simulations. The Canadian Journal of Chemical Engineering, 2003. 81(3-4): p. 692-706.

25. Becker, S., A. Sokolichin, and G. Eigenberger, Gas-liquid flow in bubble columns and loop reactors: Part II. Comparison of detailed experiments and flow simulations. Chemical Engineering Science, 1994. 49(24): p. 5747-5762.

26. Pfleger, D., et al., Hydrodynamic simulations of laboratory scale bubble columns fundamental studies of the Eulerian-Eulerian modelling approach. Chemical Engineering Science, 1999. 54(21): p. 5091-5099.

27. Pan, Y., M.P. Dudukovic, and M. Chang, Numerical investigation of gas-driven flow in 2-D bubble columns. AIChE Journal, 2000. 46(3): p. 434-449.

28. Yakhot, V., et al., Development of turbulence models for shear flows by a double expansion technique. Physics of Fluids A: Fluid Dynamics, 1992. 4(7): p. 15101520.

29. Buwa, V.V. and V.V. Ranade, Dynamics of gas-liquid flow in a rectangular bubble column: experiments and single/multi-group CFD simulations. Chemical Engineering Science, 2002. 57(22): p. 4715-4736.

30. Chen, W., et al., Scale-up effects on the time-averaged and dynamic behavior in bubble column reactors. Chemical engineering science, 2001. 56(21): p. 61496155 .

31. Rampure, M.R., A.A. Kulkarni, and V.V. Ranade, Hydrodynamics of Bubble Column Reactors at High Gas Velocity: Experiments and Computational Fluid Dynamics (CFD) Simulations. Industrial \& Engineering Chemistry Research, 2007. 46(25): p. 8431-8447.

32. McClure, D.D., et al., Development of a CFD model of bubble column bioreactors: part one-a detailed experimental study. Chemical Engineering \& Technology, 2013. 36(12): p. 2065-2070. 
33. Esmaeili, A., C. Guy, and J. Chaouki, The effects of liquid phase rheology on the hydrodynamics of a gas-liquid bubble column reactor. Chemical Engineering Science, 2015. 129: p. 193-207.

34. Plais, C. and F. Augier, Effect of liquid viscosity on mixing times in bubble columns. Theoretical Foundations of Chemical Engineering, 2016. 50(6): p. 969974.

35. Kawase, Y. and M. Moo-Young, Hydrodynamics in bubble column bioreactors with fermentation broths having a yield stress. Applied Microbiology and Biotechnology, 1989. 30(6): p. 596-603.

36. Terasaka, K., et al., Oxygen transfer into xanthan solutions in partitioned bubble columns. Journal of chemical engineering of Japan, 2013. 46(1): p. 33-39.

37. McClure, D.D., et al., Development of a CFD model of bubble column bioreactors: part two-comparison of experimental data and CFD predictions. Chemical Engineering \& Technology, 2014. 37(1): p. 131-140.

38. $\mathrm{Xu}, \mathrm{L}$. , et al., Application of population balance model in the simulation of slurry bubble column. Industrial \& Engineering Chemistry Research, 2014. 53(12): p. 4922-4930.

39. CD-Adapco, STAR-CC+, in STAR-CCM+. 2017: Steve Portal.

40. Behnia, M., S. Parneix, and P.A. Durbin, Prediction of heat transfer in an axisymmetric turbulent jet impinging on a flat plate. International Journal of Heat and Mass Transfer, 1998. 41(12): p. 1845-1855.

41. Durbin, P.A., Near-wall turbulence closure modeling without "damping functions". Theoretical and Computational Fluid Dynamics, 1991. 3(1): p. 1-13.

42. Hofmann, H.M., et al., Calculations of Steady and Pulsating Impinging Jets-An Assessment of 13 Widely used Turbulence Models. Numerical Heat Transfer, Part B: Fundamentals, 2007. 51(6): p. 565-583.

43. Xing, Y., S. Spring, and B. Weigand, Experimental and Numerical Investigation of Heat Transfer Characteristics of Inline and Staggered Arrays of Impinging Jets. Journal of Heat Transfer, 2010. 132(9): p. 092201-092201. 
44. Menter, F.R., Review of the shear-stress transport turbulence model experience from an industrial perspective. International Journal of Computational Fluid Dynamics, 2009. 23(4): p. 305-316.

45. Wolfshtein, M., The velocity and temperature distribution in one-dimensional flow with turbulence augmentation and pressure gradient. International Journal of Heat and Mass Transfer, 1969. 12(3): p. 301-318.

46. Lakehal, D. and W. Rodi, Calculation of the flow past a surface-mounted cube with two-layer turbulence models. Journal of Wind Engineering and Industrial Aerodynamics, 1997. 67: p. 65-78.

47. Versteeg, H.K. and W. Malalasekera, An introduction to computational fluid dynamics: the finite volume method. 2007: Pearson Education.

48. Launder, B. and B. Sharma, Application of the energy-dissipation model of turbulence to the calculation of flow near a spinning disc. Letters in heat and mass transfer, 1974. 1(2): p. 131-137.

49. Fluent, Theory Guide, in Multiphase Flow.

50. Fluent, Users Manual, in Multiphase Flow.

51. Sokolichin, A. and G. Eigenberger, Applicability of the standard $k-\varepsilon$ turbulence model to the dynamic simulation of bubble columns: Part I. Detailed numerical simulations. Chemical Engineering Science, 1999. 54(13-14): p. 2273-2284.

52. $\mathrm{Xu}, \mathrm{L}$., et al., Numerical simulation of bubble column flows in churn-turbulent regime: comparison of bubble size models. Industrial \& Engineering Chemistry Research, 2013. 52(20): p. 6794-6802.

53. Laborde-Boutet, C., et al., CFD simulation of bubble column flows: Investigations on turbulence models in RANS approach. Chemical Engineering Science, 2009. 64(21): p. 4399-4413.

54. Chen, J., et al., Fluid dynamic parameters in bubble columns with internals. Chemical Engineering Science, 1999. 54(13): p. 2187-2197.

55. McClure, D.D., et al., Mixing in bubble column reactors: experimental study and CFD modeling. Chemical Engineering Journal, 2015. 264: p. 291-301. 